\title{
Scaled boundary FEM model for interaction of short-crested waves with a concentric porous cylindrical structure
}

\author{
Longbin Tao $^{* 1}$, M.ASCE; Hao Song ${ }^{2}$; and Subrata Chakrabarti ${ }^{3}$, F.ASCE
}

\begin{abstract}
This paper describes the development of an efficient scaled boundary finite-element model (SBFEM) for the simulation of short-crested wave interaction with a concentric porous cylindrical structure. By weakening the governing differential equation in the circumferential direction, the SBFEM is able to solve analytically the weakened equation in the radial direction. Only the cylinder boundary on the circumference of the exterior porous cylinder is discretized with curved surface finite-elements, while a complete analytical representation is obtained for the radial differential equation. Comparisons of the numerical results on wave diffraction forces and surface wave elevations at the cylinder to available analytical solutions demonstrate that excellent accuracy can be achieved by the SBFEM with a very small number of surface finite-elements. The influence of varying the wave parameters as well as the system configuration on the system hydrodynamics, including the wave force, wave run-up and diffracted wave contour is examined and extensive results on them are presented. This parametric study will help determine the various hydrodynamic effects of a concentric porous cylindrical structure.
\end{abstract}

\section{CE Database subject headings: FINITE ELEMENT METHOD; WAVE DIFFRACTION; POROUS MEDIA; CYLINDER}

\section{INTRODUCTION}

Coastal and offshore structures are often constructed with one or more protective porous layers,

\footnotetext{
${ }^{1}$ Senior Lecturer, Griffith School of Engineering, Griffith University, Queensland 4222. E-mail: 1.tao@griffith.edu.au

${ }^{2}$ Post-Doc. Research Fellow, Griffith School of Engineering, Griffith University, Queensland 4222. E-mail: h.song@griffith.edu.au

${ }^{3}$ President, Offshore Structure Analysis, Inc., Plainfield, IL60544-7096, USA. E-mail: Chakrab@aol.com
} 
in order to reduce the direct wave impact on them. Examples are rock-filled porous breakwaters outside harbors, and concentric porous outer protective structure with the main structure in its interior. One example of this application is the successful Ekofisk gravity offshore structure in the North Sea (see Fig. 1). For these reasons, wave motion through a porous structure has attracted considerable attention among researchers in coastal and ocean engineering (e.g., Vijayalakshmi et al., 2007).

Investigations on waves past a porous structure have primarily been concentrated on three engineering applications: 1) the hydrodynamic effect of a porous structure on incoming wave trains; 2) the use of a porous structure as a wavemaker; and 3) the use of a porous structure as a breakwater in a harbor (Chwang and Chan, 1998). In most cases, Darcy's law for a homogeneous porous medium has been applied.

A brief overview of the chronological development of research on porous structures is presented below. Tuck (1971) and Porter (1972) derived formal solutions using potential theory for the transmission of water waves through a thin plate with a small gap in infinite water depth. Guiney et al. (1972) extended Tuck's (1971) theory to incorporate a finite barrier thickness and obtained results, which were validated by a set of experiments. The thickness of the porous structure was found to have a pronounced effect on reducing energy transmission. Assuming normally incident waves on a long homogeneous porous structure of rectangular cross section, Madsen (1974) presented a simple explicit solution for the transmission and reflection coefficients using a linearized theory. Nasser and McCorquodale (1975) studied unsteady non-Darcy flow in rectangular rock-filled embankments with impervious cores, in which the effect of nonlinear, shallow water waves were examined through wave tank experiments.

Chwang (1983) proposed a linearized porous-wavemaker theory to analyze small-amplitude surface waves produced by horizontal oscillations of a porous vertical plate. The theory was later applied to analyze surface waves generated by a piston-type porous wavemaker near the end of a semi-infinitely long channel of constant depth (Chwang and Li, 1983). Several studies on the physical phenomenon of wave-trapping due to a porous plate or a concentric porous cylinder system 
using the porous-wavemaker theory were reported (e.g., Chwang and Dong, 1984; Faltas, 1996).

Dalrymple et al. (1991) studied the reflection and transmission of a wave train at an oblique angle of incidence by an infinitely long porous breakwater. Huang and Chao (1992) studied the inertial effect of the porous breakwater based on Biot's theory of poroelasticity. Yu and Chwang (1994a) investigated the resonance in a harbor with porous breakwaters with the wave entering at an arbitrary angle. Yu and Chwang (1994b) performed extensive study on the transmission characteristics of waves past a porous structure. The wave behavior within the porous medium was also investigated. It was found that there is an optimum thickness for a porous structure beyond which any further increase of the thickness may not lead to an appreciable improvement in reducing its transmission and reflection characteristics. Yu and Chwang (1994c) employed the boundary integral method to study wave diffraction by a horizontal porous plate submerged at a distance below the free surface in a fluid of constant depth. Chwang and Wu (1994) extended this study to wave diffraction by a porous disk. Wang and Ren (1993) also studied the performance of a flexible and porous breakwater. Additional related work can be found in the review article of Chwang and Chan (1998).

Though considerable research efforts have been devoted to the wave interaction with porous structures, relatively limited attention has been focused on the wave diffraction by a concentric bottom-mounted porous cylindrical structure, where the interior cylinder is impermeable and the exterior cylinder is thin and porous. Wang and Ren (1994) investigated analytically the plane wave diffraction by the above-mentioned system. They found that hydrodynamic forces on the interior cylinder as well as wave amplitudes around the windward side of the interior cylinder are reduced when compared to the case of a direct wave impact on the interior cylinder. As the annular spacing increases, the hydrodynamic force on the interior cylinder decreases. It was further shown that, as the porosity of the exterior cylinder increases, the hydrodynamic force on the interior cylinder increases. Li et al. (2003) reported similar results from their experimental and numerical study with only partial porosity in the circumferential direction of the exterior cylinder. Darwiche et al. (1994) studied the wave diffraction by a two-cylinder system, with the exterior cylinder being 
porous only in the vicinity of free surface. Williams and Li (1998) further extended the work by mounting the interior cylinder on a storage tank. Zhong and Wang (2006) obtained solitary wave solutions for a concentric porous cylinder system.

The understanding of the problem, however, is still far from being complete. One of the most noticeable limitations in the previous studies is the two-dimensional plane wave assumption. It is noted that wind-generated waves in real oceans are much better represented by short-crested waves (3D) than by plane waves (2D) (Zhu, 1993; Zhu and Moule, 1994). Multi-directional waves may also arise, from the oblique interaction of two travelling plane waves or intersecting swell waves, from the reflection of waves at non-normal incidence off a vertical seawall or a breakwater, as well as from diffraction about the surface boundaries of a structure of finite length (Fuhrman and Madsen, 2006). Such waves are of paramount importance in an engineering design. Unlike the plane waves propagating in a single direction, and the standing waves fluctuating vertically in a confined region, short-crested waves can be doubly periodic in two horizontal directions, one in the direction of propagation and the other normal to it (Tsai et al., 1994).

Zhu (1993) studied the diffraction problem for a circular cylinder in short-crested waves using linear potential wave theory and found that the pressure distribution and wave run-up on the cylinder were quite different from those of plane incident waves. Their patterns become very complex as $k a$ (i.e., total incident wave number $k$ times cylinder radius $a$.) becomes large. The hydrodynamic forces on the cylinder become smaller as the spreading of the incident short-crested waves increases. Subsequently, Zhu and Moule (1994) observed that the hydrodynamic force induced by short-crested waves varies with the phasing of the waves along the spreading angle.

However, research on the short-crested wave interaction with a concentric porous cylindrical structure has been relatively few. Moreover, questions may arise whether the conclusions drawn by Zhu (1993) is still valid for a porous concentric cylindrical structure. Also, does the free surface in the annular space remain short-crested and what about the lee region?

The present study aims at answering these questions in a quantitative manner by developing a new numerical model. Recently, a semi-analytical method, called scaled boundary finite-element 
method (SBFEM) for solving linear partial differential equations has found successful application to soil-structure interaction problems. The SBFEM method was proposed by Song and Wolf (1997) and systematically described by Wolf (2003). Combining the distinct advantages of the finite-element and boundary-element methods (BEM), only the structure boundary is discretized with surface finite-elements. This, in turn, transforms the governing partial differential equations to a set of ordinary differential equations, and solves them analytically. The method represents singularities and unbounded domains accurately and efficiently when compared to the complete finite-element method and requires no fundamental solution as needed by the boundary-element method. Fewer elements are required to obtain very accurate results (Wolf, 2003).

Deeks and Cheng (2003) developed a scaled boundary finite-element solution to the twodimensional uniform potential flow around obstacles and revealed its inherent ability and advantages to model unbounded fluid problems, as well as, the singular points in the near field of bluff obstacles.

Only recently has the SBFEM been applied to wave diffraction in which the radiation condition at infinity is required to be satisfied by both the incident and scattered waves. Li et al. (2004) attempted to solve the problem of plane wave diffraction by a vertical cylinder using SBFEM with only limited success in obtaining semi-analytical solution for high frequency waves. Similar to the approach of Wolf (2003) in obtaining a solution for soil-structure interaction, Li et al. (2004) adopted a power series in the form $\left(\sum_{m=0}^{+\infty} \mathbf{C}_{m} \bar{\xi}^{-m}\right)$. Since the solution obtained as asymptotic expansions involves sums to infinity, for large values of $\bar{\xi}$ the series approaches the exact solution rapidly and only a few terms in the series need to be computed. However, this is only the case at the cylinder boundary $(\bar{\xi}=k a$, where $k$ and $a$ denote the wave number and the radius of the cylinder respectively) for high frequency waves. For low frequency waves, the series converges very slowly, hardly approaching the exact solution . Therefore, additional numerical methods, such as, the Runge-Kutta scheme, were proposed to solve the radial differential equation for low frequency waves. Such compromised approach, however, significantly diminishes the advantages of SBFEM exhibited in dealing with bounded fluid domain problems. 
Tao et al. (2007) applied the SBFEM to solve wave interaction with a single circular cylinder. Instead of using a power series, Tao et al. (2007) chose Hankel function to solve the Helmholtz equation in the unbounded domain. The radial differential equation is solved fully analytically in all frequency ranges. Without relying on any other numerical schemes, the semi-analytical model for the plane-wave diffraction by a single circular cylinder is shown to reproduce the analytical solution for all the physical properties including wave run-up, effective inertia and drag coefficients, and total force very accurately and at very low computational cost.

This paper develops a SBFEM model for simulations of short-crested wave interaction with a concentric porous cylindrical structure. The presence of the wave-structure interaction in both the bounded and unbounded domains imposes particular challenges to the numerical model development. The following section provides description of the governing equations and summarizes it for the particular physical problem. Following this section, the transformation of the above governing scaled boundary finite-element equations and the corresponding boundary conditions are presented and the detailed solution techniques are discussed. Only a few finite elements discretized on the circumference of the cylinders are shown to be sufficient to obtain accurate results. Finally, detailed numerical results on wave forces and surface elevations over a broad range of incident short-crested wave parameters as well as structure configurations including the porosity of the exterior cylinder and the annular gap between the two cylinders are presented.

\section{MATHEMATICAL FORMULATION}

Consider a monochromatic short-crested wave train propagating in the direction of the positive $x$ axis. A structure consisting of two concentric fixed vertical cylinders extend from the sea bottom to above the free surface of the ocean along $z$ axis. The origin is placed at the center of the cylinders on the mean water surface (see Fig. 2). The exterior cylinder is made porous and the interior cylinder is impermeable. The fluid domain is divided into two regions, the annular region $\Omega_{1}$ and the region of the outside of the exterior cylinder $\Omega_{2}$. The following notation have been used in the paper: $\Phi_{j}=$ total velocity potential, $\Phi^{I}=$ velocity potential of incident wave, $\Phi^{S}=$ velocity potential of scattered wave, $k=$ total wave number, $k_{x}=$ wave number in $x$ direction, $k_{y}=$ wave 
where

$$
\nabla^{2} \Phi_{j}(x, z)=0 \quad \text { in } \quad \Omega_{j},
$$

subject to the combined free surface boundary condition

$$
\Phi_{j, t t}+g \Phi_{j, z}=0 \quad \text { at } \quad z=0
$$

and the bottom condition

$$
\Phi_{j, z}=0 \quad \text { at } \quad z=-h,
$$

where the comma in the subscript designates partial derivative with respect to the variable following the comma.

The velocity potentials can be decomposed by separating the vertical variable $z$ and the time $t$ from each component as

$$
\begin{array}{ll}
\Phi_{j}(x, y, z, t)=\phi_{j}(x, y) Z(z) e^{-i \omega t} & \text { in } \quad \Omega_{j}, \\
\Phi_{2}^{I}(x, y, z, t)=\phi_{2}^{I}(x, y) Z(z) e^{-i \omega t} & \text { in } \quad \Omega_{2}, \\
\Phi_{2}^{S}(x, y, z, t)=\phi_{2}^{S}(x, y) Z(z) e^{-i \omega t} & \text { in } \quad \Omega_{2},
\end{array}
$$




$$
Z(z)=\frac{\cosh k(z+h)}{\cosh k h}
$$

This procedure leads to the sea bottom condition being automatically satisfied, and the linearized free surface boundary condition is satisfied using the following dispersion relationship

$$
\omega^{2}=g k \tanh k h
$$

The relationship between the total velocity potential, and the scattered, and incident wave velocity potentials is

$$
\Phi_{2}=\Phi_{2}^{I}+\Phi_{2}^{S}, \quad \phi_{2}=\phi_{2}^{I}+\phi_{2}^{S} \quad \text { in } \quad \Omega_{2} .
$$

Taylor (1956) showed that the fluid flow passing through the porous boundary can be essentially assumed to obey Darcy's law if the boundary is made of fine pores. Hence, the porous flow velocity is linearly proportional to the pressure difference between the two sides of the porous boundary, and the boundary condition on exterior porous cylinder can be expressed as

$$
\phi_{1, n}=-\phi_{2, n}=i G_{0} k\left(\phi_{1}-\phi_{2}\right) \quad \text { on } \quad r=b,
$$

where $G_{0}=\frac{\rho \omega d}{\mu k}$ (Chwang and Li, 1983), is a measure of the porous effect, $\mu$ is the coefficient of dynamic viscosity, $d$ is a material constant having the dimension of a length, and $n$ denotes the normal to the boundary. Such an approach was firstly applied to wave study by Chwang (1983) and subsequently by many other researchers.

Thus, the problem becomes to two-dimensional at the free surface. The function $\phi_{2}^{S}(x, y)$ in $\Omega_{2}$ is governed by the Helmholtz equation with the boundary condition at the interface of fluid and porous cylinder, and the radiation condition at infinity, namely, the Sommerfeld condition as follows: 


$$
\begin{gathered}
\nabla^{2} \phi_{2}^{S}+k^{2} \phi_{2}^{S}=0 \quad \text { in } \quad \Omega_{2}, \\
\phi_{2, n}^{S}=-i G_{0} k\left(\phi_{1}-\phi_{2}^{S}-\phi_{2}^{I}\right)-\phi_{2, n}^{I} \quad \text { on } \quad r=b, \\
\lim _{k r \rightarrow \infty}(k r)^{1 / 2}\left(\phi_{2, r}^{S}-i k \phi_{2}^{S}\right)=0 \quad \text { in } \quad \Omega_{2},
\end{gathered}
$$

where $r$ is the radial axis, and $i=\sqrt{-1}$.

The function $\phi_{1}(x, y)$ in $\Omega_{1}$ is governed by the Helmholtz equation with the boundary conditions at the interface of fluid and interior solid cylinder at $r=a$ and exterior porous cylinder at $r=b$ :

$$
\begin{gathered}
\nabla^{2} \phi_{1}+k^{2} \phi_{1}=0 \quad \text { in } \Omega_{1}, \\
\phi_{1, n}=0 \quad \text { on } \quad r=a, \\
\phi_{1, n}=i G_{0} k\left(\phi_{1}-\phi_{2}^{S}-\phi_{2}^{I}\right) \quad \text { on } \quad r=b .
\end{gathered}
$$

The velocity potential of the linear short-crested incident wave (Fuchs, 1952) travelling principally in the positive $x$ direction is given by the real part of

$$
\Phi_{I}=-\frac{i g A}{\omega} Z(z) e^{i\left(k_{x} x-\omega t\right)} \cos \left(k_{y} y\right) \quad \text { in } \quad \Omega_{2} .
$$

Eqs. (11)-(16) constitute two sets of the governing equation and boundary conditions for the diffraction of short-crested waves by concentric vertical porous cylindrical structure, corresponding to boundary value problems in a bounded domain and an unbounded domain respectively. After obtaining $\phi_{2}^{S}, \Phi_{2}$ and $\Phi_{1}$ by solving the above boundary-value problems, the velocity, free surface 
elevation and the dynamic pressure can be calculated respectively from

$$
\begin{gathered}
\mathbf{v}_{j}=\nabla \Phi_{j}, \\
\eta_{j}=\frac{i \omega}{g} \phi_{j}, \\
p_{j}=-\rho \Phi_{j, t} .
\end{gathered}
$$

\section{SCALED BOUNDARY FINITE-ELEMENT TRANSFORMATION}

In this section, $\phi_{1}$ and $\phi_{2}^{S}$ will both be denoted as $\phi$ for brevity, and the region $\Omega_{j}$ will be denoted as $\Omega$. If the velocity boundary is defined by $\Gamma_{v}$, we have

$$
\phi_{, n}=\bar{v}_{n}, \quad \text { on } \quad \Gamma_{v},
$$

where the overbar denotes a prescribed value.

The finite-element method requires the weighted residuals of the governing equation to be zero. Hence Eqs. (11), (14) and (21) are multiplied by a weighting function $w$ and integrated over the flow domain and the boundary. Performing integration by parts, the resulting equation becomes

$$
\int_{\Omega} \nabla^{T} w \nabla \phi d \Omega-\int_{\Omega} w k^{2} \phi d \Omega-\oint_{\Gamma} w \bar{v}_{n} d \Gamma=0 .
$$

SBFEM defines the domain $\Omega$ by scaling a single piecewise-smooth curve $S$ relative to a scaling center $\left(x_{0}, y_{0}\right)$, which is chosen at the cylinder center in this case (see Fig. 3). The circumferential coordinate $s$ is anticlockwise along the curve $S$ and the normalized radial coordinate $\xi$ is a scaling factor, defined as 1 at curve $S$ and 0 at the scaling center. The whole solution domain $\Omega$ is in the range of $\xi_{0} \leq \xi \leq \xi_{1}$ and $s_{0} \leq s \leq s_{1}$. The two straight sections $s=s_{0}$ and $s=s_{1}$ are called side-faces. They coincide, if the curve $S$ is closed. For bounded domain, $\xi_{0}=0$ and $\xi_{1}=1$; 
whereas, for unbounded domain, $\xi_{0}=1$ and $\xi_{1}=\infty$. Therefore the Cartesian coordinates are transformed to the scaled boundary coordinate $\xi$ and $s$ with the scaling equations

$$
x=x_{0}+\xi x_{s}(s), \quad y=y_{0}+\xi y_{s}(s) .
$$

By employing SBFEM, an approximate solution of $\phi$ is sought as

$$
\phi_{A}(\xi, s)=\mathbf{N}(s) \mathbf{a}(\xi),
$$

where $\mathbf{N}(s)$ is the shape function, the vector $\mathbf{a}(\xi)$ is analogous to the nodal values same as in FEM. The radial function $a_{j}(\xi)$ represents the variation of the scattered wave potential in the radial axis $\xi$ at each node $j$, and the shape function $\mathbf{N}(s)$ interpolates between the nodal potential values in the circumferential axis $s$. The separation of variable technique has its limitation in terms of boundary surfaces (Morse and Feshbach, 1953), since a completely general boundary surface will have the value of A varying both as functions of $\xi$ and $s$ and these two variables may not be separated with independent functions. However, for many axisymmetric structures, e.g., which are indeed of interest for offshore applications, this technique will work well in which the two variables $\xi$ and $s$ may be separated as shown in Eq. (24).

By performing scaled boundary transformation, the operator $\nabla$ can be expressed as (Wolf, 2003):

$$
\nabla=\mathbf{b}_{1}(s) \frac{\partial}{\partial \xi}+\frac{1}{\xi} \mathbf{b}_{2}(s) \frac{\partial}{\partial s},
$$

where $\mathbf{b}_{1}(s)$ and $\mathbf{b}_{2}(s)$ are dependent only on the boundary definition

$$
\mathbf{b}_{1}(s)=\frac{1}{|J|}\left\{\begin{array}{c}
y_{s}(s)_{, s} \\
-x_{s}(s)_{, s}
\end{array}\right\}, \quad \mathbf{b}_{2}(s)=\frac{1}{|J|}\left\{\begin{array}{c}
-y_{s}(s) \\
x_{s}(s)
\end{array}\right\},
$$

and $|J|$ is the Jacobian at the boundary 


$$
|J|=x_{s}(s) y_{s}(s)_{, s}-y_{s}(s) x_{s}(s)_{, s} .
$$

226 From Eqs. (18) and (25), the approximate velocity can be expressed as

$$
v_{A}(\xi, s)=\mathbf{B}_{1}(s) \mathbf{a}(\xi)_{, \xi}+\frac{1}{\xi} \mathbf{B}_{2}(s) \mathbf{a}(\xi),
$$

227 where

$$
\mathbf{B}_{1}(s)=\mathbf{b}_{1}(s) \mathbf{N}(s), \quad \mathbf{B}_{2}(s)=\mathbf{b}_{2}(s) \mathbf{N}(s)_{, s} .
$$

Applying the Galerkin approach, the weighting function $w$ can be formulated using the same 229 shape function as in Eq. (24)

$$
w(\xi, s)=\mathbf{N}(s) \mathbf{w}(\xi)=\mathbf{w}(\xi)^{T} \mathbf{N}(s)^{T} .
$$

230

Substituting Eqs. (24), (25), (29) and (30) into Eq. (22) results in

$$
\begin{aligned}
& \int_{\Omega}\left[\mathbf{B}_{1}(s) \mathbf{w}(\xi)_{, \xi}+\frac{1}{\xi} \mathbf{B}_{2}(s) \mathbf{w}(\xi)\right]^{T}\left[\mathbf{B}_{1}(s) \mathbf{a}(\xi)_{, \xi}+\frac{1}{\xi} \mathbf{B}_{2}(s) \mathbf{a}(\xi)\right] d \Omega \\
& -\int_{\Omega} k^{2} \mathbf{w}(\xi)^{T} \mathbf{N}(s)^{T} \mathbf{N}(s) \mathbf{a}(\xi) d \Omega-\oint_{\Gamma} \mathbf{w}(\xi)^{T} \mathbf{N}(s)^{T} \bar{v}_{n} d \Gamma=0,
\end{aligned}
$$

where the incremental volume (Wolf, 2003) is

$$
d \Omega=|J| \xi d \xi d s .
$$

232 For convenience, coefficient matrices are introduced here as 


$$
\begin{aligned}
\mathbf{E}_{0} & =\int_{S} \mathbf{B}_{1}(s)^{T} \mathbf{B}_{1}(s)|J| d s \\
\mathbf{E}_{1} & =\int_{S} \mathbf{B}_{2}(s)^{T} \mathbf{B}_{1}(s)|J| d s \\
\mathbf{E}_{2} & =\int_{S} \mathbf{B}_{2}(s)^{T} \mathbf{B}_{2}(s)|J| d s \\
\mathbf{M}_{0} & =\int_{S} \mathbf{N}(s)^{T} \mathbf{N}(s)|J| d s \\
\mathbf{F}_{s}(\xi) & =\mathbf{N}\left(s_{0}\right)^{T}\left(-\bar{v}_{n}\left(\xi, s_{0}\right)\right)\left|J\left(s_{0}\right)\right|+\mathbf{N}\left(s_{1}\right)^{T}\left(-\bar{v}_{n}\left(\xi, s_{1}\right)\right)\left|J\left(s_{1}\right)\right| .
\end{aligned}
$$

The above integrals Eqs. (33)-(36) can be computed element by element and assembled together for the entire boundary. Expanding Eq. (31) and integrating the terms containing $\mathbf{w}(\xi)_{, \xi}$ by parts with respect to $\xi$ using Green's theorem leads to

$$
\begin{aligned}
& \mathbf{w}\left(\xi_{1}\right)^{T}\left[\mathbf{E}_{0} \xi_{1} \mathbf{a}\left(\xi_{1}\right)_{, \xi}+\mathbf{E}_{1}^{T} \mathbf{a}\left(\xi_{1}\right)-\int_{S} \mathbf{N}(s)^{T}\left(\bar{v}_{n}\left(\xi_{1}, s\right)\right) \xi_{1} d s\right] \\
- & \mathbf{w}\left(\xi_{0}\right)^{T}\left[\mathbf{E}_{0} \xi_{0} \mathbf{a}\left(\xi_{0}\right)_{, \xi}+\mathbf{E}_{1}^{T} \mathbf{a}\left(\xi_{0}\right)+\int_{S} \mathbf{N}(s)^{T}\left(\bar{v}_{n}\left(\xi_{0}, s\right)\right) \xi_{0} d s\right] \\
- & \int_{\xi_{0}}^{\xi_{1}} \mathbf{w}(\xi)^{T}\left[\mathbf{E}_{0} \xi \mathbf{a}(\xi)_{, \xi \xi}+\left(\mathbf{E}_{0}+\mathbf{E}_{1}^{T}-\mathbf{E}_{1}\right) \mathbf{a}(\xi)_{, \xi}-\mathbf{E}_{2} \frac{1}{\xi} \mathbf{a}(\xi)+k^{2} \xi \mathbf{M}_{0} \mathbf{a}(\xi)-\mathbf{F}_{s}(\xi)\right] d \xi \\
& =0 .
\end{aligned}
$$

To satisfy all sets of weighting function $\mathbf{w}(\xi)$, the following conditions must be satisfied:

$$
\begin{gathered}
\mathbf{E}_{0} \xi_{1} \mathbf{a}\left(\xi_{1}\right)_{, \xi}+\mathbf{E}_{1}^{T} \mathbf{a}\left(\xi_{1}\right)=\int_{S} \mathbf{N}(s)^{T}\left(\bar{v}_{n}\left(\xi_{1}, s\right)\right) \xi_{1} d s \\
\mathbf{E}_{0} \xi_{0} \mathbf{a}\left(\xi_{0}\right)_{, \xi}+\mathbf{E}_{1}^{T} \mathbf{a}\left(\xi_{0}\right)=-\int_{S} \mathbf{N}(s)^{T}\left(\bar{v}_{n}\left(\xi_{0}, s\right)\right) \xi_{0} d s \\
\mathbf{E}_{0} \xi^{2} \mathbf{a}(\xi)_{, \xi \xi}+\left(\mathbf{E}_{0}+\mathbf{E}_{1}^{T}-\mathbf{E}_{1}\right) \xi \mathbf{a}(\xi)_{, \xi}-\mathbf{E}_{2} \mathbf{a}(\xi)+k^{2} \xi^{2} \mathbf{M}_{0} \mathbf{a}(\xi)=\xi \mathbf{F}_{s}(\xi) .
\end{gathered}
$$

Eq. (41) is the so-called scaled boundary finite-element equation. By introducing the shape 
function, the Helmholtz equation has been weakened in the circumferential direction, so that the governing partial differential equation is transformed to an ordinary matrix differential equation in radial direction. The rank of matrices $\mathbf{E}_{0}, \mathbf{E}_{1}, \mathbf{E}_{2}, \mathbf{M}_{0}$ and vector $\mathbf{a}(\xi)$ is $m$ (where $m$ is the number of nodes in the curve $S$ ). In the present study, the side-faces coincide so that the flow across the side-faces is equal and opposite, leading to vanishing of the term $\mathbf{F}_{s}(\xi)$. Therefore, the final governing equation, Eq. (41), is a homogeneous second-order ordinary matrix differential equation in terms of matrix of rank $m$.

Boundary conditions, Eqs. (12) and (13) or Eqs. (15) and (16), are weakened in the form of Eqs. (40) and (39) respectively, indicating the relationship between the integrated nodal flow on the boundary and the velocity potentials of the nodes. For the wave diffraction problem in the unbounded region $\Omega_{2}, \xi_{0}=1$ on the boundary of exterior porous cylinder and $\xi_{1}=+\infty$ at infinity. For the boundary-value problem in the bounded annular region $\Omega_{1}, \xi_{0}=0$ and $\xi_{1}=1$.

\section{SOLUTION PROCEDURE}

For the exterior porous cylinder, we have

$$
x_{s}(s)=b \cos (s / b), \quad y_{s}(s)=b \sin (s / b)
$$

From Eqs. (23), (26), (27), (29) and (33)-(36), $x_{s}(s)_{, s}, y_{s}(s)_{, s}, \mathbf{b}_{1}(s), \mathbf{b}_{2}(s),|J|, \mathbf{B}_{1}(s), \mathbf{B}_{2}(s)$,

$\mathbf{E}_{0}, \mathbf{E}_{1}, \mathbf{E}_{2}$, and $\mathbf{M}_{0}$ can be calculated accordingly. The following relationships hold:

$$
\begin{gathered}
\mathbf{E}_{1}=0 \cdot \mathbf{I}, \quad \mathbf{E}_{0}^{-1} \mathbf{M}_{0}=b^{2} \mathbf{I}, \\
\mathbf{E}_{0}=\frac{1}{b} \int_{S} \mathbf{N}(s)^{T} \mathbf{N}(s) d s
\end{gathered}
$$

where $\mathbf{I}$ is the identity matrix of rank $m$.

Using Eq. (43), pre-multiplying both sides of Eq. (41) by $\mathbf{E}_{0}^{-1}$ and simplifying, we have

$$
\zeta^{2} \mathbf{a}(\zeta)_{, \zeta \zeta}+\zeta \mathbf{a}(\zeta)_{, \zeta}-\mathbf{E}_{0}^{-1} \mathbf{E}_{2} \mathbf{a}(\zeta)+\zeta^{2} \mathbf{a}(\zeta)=0
$$




$$
\zeta=k b \xi
$$

\section{Solution for unbounded domain $\Omega_{2}$}

Eq. (45) is the matrix form of Bessel's differential equation. Considering the Sommerfeld radiation condition Eq. (13), it is logical to select $H_{r_{j}}(\zeta) \mathbf{T}_{j}$ as a base solution of Eq. (45) in region $\Omega_{2}$

The solution for $\mathbf{a}_{2}(\zeta)$ is then expressed in the series form:

$$
\mathbf{a}_{2}(\zeta)=\sum_{j=1}^{m} c_{j} H_{r_{j}}(\zeta) \mathbf{T}_{j}=\mathbf{T H}(\zeta) \mathbf{C},
$$

where $\mathbf{T}_{j}$ are vectors of rank $m, c_{j}$ are coefficients, $H_{r_{j}}(\zeta)$ are the Hankel functions of the first kind, and

$$
\begin{aligned}
\mathbf{T} & =\left[\mathbf{T}_{1}, \mathbf{T}_{2}, \cdots, \mathbf{T}_{m}\right], \\
\mathbf{H}(\zeta) & =\operatorname{diag}\left[H_{r_{1}}(k b \xi), H_{r_{2}}(k b \xi), \cdots, H_{r_{m}}(k b \xi)\right], \\
\mathbf{C} & =\left[c_{1}, c_{2}, \cdots, c_{m}\right]^{T},
\end{aligned}
$$

where "diag" denotes a diagonal matrix with the elements in the square brackets on the main diagonal.

Substituting Eq. (47) into Eq. (45), and using the following properties of Hankel function

$$
\begin{aligned}
\zeta^{2} H_{r_{j}}^{\prime \prime}(\zeta) & =-\zeta^{2} H_{r_{j}}(\zeta)+\zeta H_{r_{j}+1}(\zeta)-r_{j} H_{r_{j}}(\zeta)+r_{j}^{2} H_{r_{j}}(\zeta), \\
\zeta H_{r_{j}}^{\prime}(\zeta) & =-\zeta H_{r_{j}+1}(\zeta)+r_{j} H_{r_{j}}(\zeta),
\end{aligned}
$$


where the prime and the double prime denote the first and second derivatives with respect to the argument $\zeta$ respectively, we have

$$
\sum_{j=1}^{m}\left(\mathbf{E}_{0}^{-1} \mathbf{E}_{2}-r_{j}^{2} \mathbf{I}\right) \mathbf{T}_{j} \cdot c_{j} H_{r_{j}}(\zeta)=0 .
$$

For any $c_{j} H_{r_{j}}(\zeta)$, Eq. (53) yields

$$
\left(\mathbf{E}_{0}^{-1} \mathbf{E}_{2}-r_{j}^{2} \mathbf{I}\right) \mathbf{T}_{j}=0
$$

Let $\lambda_{j}$ be the eigenvalues of $\mathbf{E}_{0}^{-1} \mathbf{E}_{2}$, then $r_{j}=\sqrt{\lambda_{j}}$, and $\mathbf{T}_{j}$ are the eigenvectors of $\mathbf{E}_{0}^{-1} \mathbf{E}_{2}$.

Since the Sommerfeld radiation condition (13) or (39) has been satisfied by the Hankel functions, we now only consider the body boundary condition (40) of the circular cylinder

$$
\mathbf{E}_{0} k b \sum_{j=1}^{m} c_{j} H_{r_{j}}^{\prime}(k b) \mathbf{T}_{j}=-\left[\int_{S} \mathbf{N}(s)^{T} \mathbf{N}(s) d s\right] \overline{\mathbf{v}}_{2 n}^{S},
$$

where $\overline{\mathbf{v}}_{2 n}^{S}$ is the vector of nodal normal velocity of scattered wave in region $\Omega_{2}$ on the body boundary.

\section{Solution for bounded domain $\Omega_{1}$}

Similar approach is applied to the region $\Omega_{1}$. Assume

$$
\mathbf{a}_{1}(\zeta)=\sum_{j=1}^{m}\left[c_{j}^{1} J_{r_{j}}(\zeta)+c_{j}^{2} Y_{r_{j}}(\zeta)\right] \mathbf{T}_{j}=\mathbf{T}\left[\mathbf{J}(\zeta) \mathbf{C}^{1}+\mathbf{Y}(\zeta) \mathbf{C}^{2}\right]
$$

where $c_{j}^{1}$ and $c_{j}^{2}$ are coefficients, $J_{r_{j}}(\zeta)$ are the Bessel functions of the first kind, $Y_{r_{j}}(\zeta)$ are the Bessel functions of the second kind, and 


$$
\begin{aligned}
\mathbf{C}^{1} & =\left[c_{1}^{1}, c_{2}^{1}, \cdots, c_{m}^{1}\right]^{T}, \\
\mathbf{C}^{2} & =\left[c_{1}^{2}, c_{2}^{2}, \cdots, c_{m}^{2}\right]^{T}, \\
\mathbf{J}(\xi) & =\operatorname{diag}\left[J_{r_{1}}(k b \xi), J_{r_{2}}(k b \xi), \cdots, J_{r_{m}}(k b \xi)\right], \\
\mathbf{Y}(\xi) & =\operatorname{diag}\left[Y_{r_{1}}(k b \xi), Y_{r_{2}}(k b \xi), \cdots, Y_{r_{m}}(k b \xi)\right] .
\end{aligned}
$$

Again if $\lambda_{j}$ is the eigenvalues of $\mathbf{E}_{0}^{-1} \mathbf{E}_{2}$, then $r_{j}=\sqrt{\lambda_{j}}$, and $\mathbf{T}$ is the eigenvector of $\mathbf{E}_{0}^{-1} \mathbf{E}_{2}$. Applying boundary conditions on the interior cylinder Eq. (40) and the exterior porous cylinder Eq. (39), respectively, we have

$$
\begin{gathered}
\mathbf{C}^{2}=-\mathbf{Y}_{a}^{\prime-1} \mathbf{J}_{a}^{\prime} \mathbf{C}^{1}, \\
\mathbf{E}_{0} k b \mathbf{T}\left(\mathbf{J}_{b}^{\prime} \mathbf{C}^{1}+\mathbf{Y}_{b}^{\prime} \mathbf{C}^{2}\right)=\left[\int_{S} \mathbf{N}(s)^{T} \mathbf{N}(s) d s\right] \overline{\mathbf{v}}_{1 n},
\end{gathered}
$$

where $\overline{\mathbf{v}}_{1 n}$ is the vector of nodal total normal velocity in region $\Omega_{1}$ on the body boundary of exterior cylinder.

Combining Eqs. (10), (21), (24), (44), (47), (55), (56) and (62), and noting

$$
\overline{\mathbf{v}}_{2 n}^{I}+\overline{\mathbf{v}}_{2 n}^{S}=\overline{\mathbf{v}}_{2 n}=-\overline{\mathbf{v}}_{1 n}
$$

where $\overline{\mathbf{v}}_{2 n}^{I}$ is the vector of nodal normal velocity of incident wave in the region $\Omega_{2}$ on the body boundary of the exterior cylinder, $\mathbf{a}_{1}(\xi)$ and $\mathbf{a}_{2}(\xi)$ are solved as

$$
\begin{aligned}
& \mathbf{a}_{1}(\xi)=\mathbf{T}\left[\mathbf{J}(\xi) \mathbf{Y}_{a}^{\prime}-\mathbf{Y}(\xi) \mathbf{J}_{a}^{\prime}\right] \mathbf{W}^{-1}\left(\mathbf{T}^{-1} \overline{\mathbf{a}}_{2}^{I}+\mathbf{H}_{b h} \mathbf{T}^{-1} \overline{\mathbf{v}}_{2 n}^{I} / k\right), \\
& \mathbf{a}_{2}(\xi)=\mathbf{T} \mathbf{H}_{h}(\xi) \mathbf{W}^{-1}\left[\left(\mathbf{J}_{b}^{\prime} \mathbf{Y}_{a}^{\prime}-\mathbf{Y}_{b}^{\prime} \mathbf{J}_{a}^{\prime}\right) \mathbf{T}^{-1} \overline{\mathbf{a}}_{2}^{I}+\mathbf{V} \mathbf{T}^{-1} \overline{\mathbf{v}}_{2 n}^{I} / k\right],
\end{aligned}
$$




$$
\begin{aligned}
\mathbf{V} & =-\frac{1}{i G}\left(\mathbf{J}_{b}^{\prime} \mathbf{Y}_{a}^{\prime}-\mathbf{Y}_{b}^{\prime} \mathbf{J}_{a}^{\prime}\right)+\left(\mathbf{J}_{b} \mathbf{Y}_{a}^{\prime}-\mathbf{Y}_{b} \mathbf{J}_{a}^{\prime}\right), \\
\mathbf{W} & =\mathbf{V}-\mathbf{H}_{b h}\left(\mathbf{J}_{b}^{\prime} \mathbf{Y}_{a}^{\prime}-\mathbf{Y}_{b}^{\prime} \mathbf{J}_{a}^{\prime}\right),
\end{aligned}
$$

and

$$
\begin{aligned}
\mathbf{J}_{a}^{\prime} & =\operatorname{diag}\left[J_{r_{1}}^{\prime}(k a), J_{r_{2}}^{\prime}(k a), \cdots, J_{r_{m}}^{\prime}(k a)\right], \\
\mathbf{Y}_{a}^{\prime} & =\operatorname{diag}\left[Y_{r_{1}}^{\prime}(k a), Y_{r_{2}}^{\prime}(k a), \cdots, Y_{r_{m}}^{\prime}(k a)\right], \\
\mathbf{J}_{b} & =\operatorname{diag}\left[J_{r_{1}}(k b), J_{r_{2}}(k b), \cdots, J_{r_{m}}(k b)\right], \\
\mathbf{Y}_{b} & =\operatorname{diag}\left[Y_{r_{1}}(k b), Y_{r_{2}}(k b), \cdots, Y_{r_{m}}(k b)\right], \\
\mathbf{J}_{b}^{\prime} & =\operatorname{diag}\left[J_{r_{1}}^{\prime}(k b), J_{r_{2}}^{\prime}(k b), \cdots, J_{r_{m}}^{\prime}(k b)\right], \\
\mathbf{Y}_{b}^{\prime} & =\operatorname{diag}\left[Y_{r_{1}}^{\prime}(k b), Y_{r_{2}}^{\prime}(k b), \cdots, Y_{r_{m}}^{\prime}(k b)\right],
\end{aligned}
$$

$$
\begin{aligned}
\mathbf{H}_{h}(\xi) & =\operatorname{diag}\left[H_{r_{1}}(k b \xi) / H_{r_{1}}^{\prime}(k b), H_{r_{2}}(k b \xi) / H_{r_{2}}^{\prime}(k b), \cdots, H_{r_{m}}(k b \xi) / H_{r_{m}}^{\prime}(k b)\right], \\
\mathbf{H}_{b h} & =\operatorname{diag}\left[H_{r_{1}}(k b) / H_{r_{1}}^{\prime}(k b), H_{r_{2}}(k b) / H_{r_{2}}^{\prime}(k b), \cdots, H_{r_{m}}(k b) / H_{r_{m}}^{\prime}(k b)\right] .
\end{aligned}
$$
$\mathbf{a}_{1}(\xi)$ and $\mathbf{a}_{2}(\xi)$ are

$$
\begin{gathered}
\mathbf{a}_{1}(\xi)=\mathbf{T} \mathbf{J}(\xi) \mathbf{W}^{-1}\left(\mathbf{T}^{-1} \overline{\mathbf{a}}_{2}^{I}+\mathbf{H}_{b h} \mathbf{T}^{-1} \overline{\mathbf{v}}_{2 n}^{I} / k\right), \\
\mathbf{a}_{2}(\xi)=\mathbf{T H}{ }_{h}(\xi) \mathbf{W}^{-1}\left(\mathbf{J}_{b}^{\prime} \mathbf{T}^{-1} \overline{\mathbf{a}}_{2}^{I}+\mathbf{V} \mathbf{T}^{-1} \overline{\mathbf{v}}_{2 n}^{I} / k\right),
\end{gathered}
$$




$$
\begin{aligned}
\mathbf{V} & =-\frac{1}{i G} \mathbf{J}_{b}^{\prime}+\mathbf{J}_{b}, \\
\mathbf{W} & =\mathbf{V}-\mathbf{H}_{b h} \mathbf{J}_{b}^{\prime} .
\end{aligned}
$$

$$
\frac{d F_{x}}{d z}=-R \int_{0}^{2 \pi} p \cdot \cos (\theta) d \theta=2 \pi R P\left(k_{x}, k_{y}, k, R\right) \cdot \rho g A \cdot Z(z) e^{-i \omega t}
$$

where the function $P\left(k_{x}, k_{y}, k, R\right)$ is the dimensionless parameter of $d F_{x} / d z$ without the term $\rho g A \cdot Z(z) e^{-i \omega t}$ and $R$ is the radius of the cylinder ( $a$ or $b$ ).

The function $P\left(k_{x}, k_{y}, k, R\right)$ determines the first-order total horizontal force in $x$ direction on the cylinder, $F_{x}$, which can be obtained by integrating Eqs. (80) with respect to $z$,

$$
F_{x}=\int_{-h}^{0} \frac{d F_{x}}{d z} d z=2 \pi R P\left(k_{x}, k_{y}, k, R\right) \cdot \rho g A e^{-i \omega t} \cdot \tanh (k h) / k .
$$

The total moment about an axis parallel to the $y$-axis passing through the bottom of the cylinder is

$$
M_{y}=\int_{-h}^{0}(z+h) \frac{d F_{x}}{d z} d z=2 \pi R P_{x}\left(k_{x}, k_{y}, k, R\right) \cdot \rho g A e^{-i \omega t} \cdot f(k h) / k^{2},
$$

where 


$$
f(k h)=[k h \tanh (k h)+\operatorname{sech}(k h)-1] .
$$

It can be concluded from Eqs. (81) and (82) that only the function $P\left(k_{x}, k_{y}, k, R\right)$ needs to be discussed. The effective inertia coefficient $C_{M}$ and linear drag coefficient $C_{D}$ per unit height are defined as

$$
R e\left(\frac{d F_{x}}{d z}\right)=\rho \pi R^{2}\left(C_{M} \dot{U}+\omega C_{D} U\right)
$$

where $U$ is the velocity of the incident waves at the origin of the cylinders in their absence and the dot represents its time derivative.

From Eqs. (17), (80) and (84), we have

$$
C_{M}=-\frac{2 P_{i}}{k_{x} R}, \quad C_{D}=\frac{2 P_{r}}{k_{x} R}
$$

where $P_{r}$ and $P_{i}$ are the real and imaginary parts of $P\left(k_{x}, k_{y}, k, R\right)$ respectively.

From Eq. (85), the total horizontal force without the constant terms can be expressed as

$$
|2 \pi R P|=\pi k_{x} R^{2} \sqrt{C_{M}^{2}+C_{D}^{2}}
$$

\section{NUMERICAL MODEL VALIDATION}

The principal steps taken to validate the present SBFEM scheme and solution procedure are (1) convergence tests using varying number of elements discretized on the surface of the cylinders; (2) comparison with the available analytical solutions (Wang and Ren, 1994) of plane-wave interaction with a concentric porous cylindrical structure; and (3) comparison with the available analytical solutions (Zhu, 1993) of short-crested waves interacting with a single circular cylinder. Taking into account the symmetry of the physical problem, only half the concentric porous structure is considered. The exterior cylinder surface is discretized with three-node quadratic elements (see Fig. 4). 


\section{Wave run-up: plane waves and short-crested waves}

Fig. 5 is a comparison of wave run-up around the interior cylinder and exterior porous cylinder, between the present SBFEM results and the analytical solutions of Wang and Ren (1994). Results shown in Fig. 5 used the same parameter values as in Wang and Ren (1994) (i.e., $h=15 \mathrm{~m}, b=10$ $\mathrm{m}, a / b=0.2, G_{0}=1.0$ and the wave-effect parameter $\left.C_{w}=g /\left(\omega^{2} h\right)=1.0\right)$. As can be seen in the figure, only two elements discretized over the cylinder boundary are sufficient to yield good agreement between the present SBFEM results and the analytical solutions.

The run-up on a single circular cylinder due to short-crested waves is shown as solid lines in Fig. 6 from the analytical solutions of Zhu (1993). In order to compare these results with the SBFEM calculation, the values of $G_{0}$ is chosen properly in its limits. The run-ups on the interior cylinder and exterior cylinder are calculated with two limiting values assigned to the porous effect $G_{0}$ for the exterior cylinder, namely, 0 and $+\infty$, respectively. When $G_{0}=0$, the exterior cylinder becomes solid, so the fluid region $\Omega_{1}$ remains at rest, and only the exterior cylinder is exposed to incident waves. On the other hand, the interior cylinder will be a single cylinder exposed to the incident wave as $G_{0}=+\infty$. Other values are chosen to match the example of Zhu (1993), i.e., $a=1 \mathrm{~m}, b=2 \mathrm{~m}, k_{x}=1 \mathrm{~m}^{-1}$ and $k_{y}=1 \mathrm{~m}^{-1}$. Again, excellent agreement with the solutions of Zhu (1993) is found where the SBFEM model with only 6 elements gave almost identical solutions to the analytical approach. Moreover, the convergence of the SBFEM scheme for wave run-up, as the number of elements discretized on the cylinder surface increases, is clearly shown in Fig. 6, and is shown to depend on the dimensionless parameters $k a$ and $k b$. The relationship between the wave run-up and $k a$ in the SBFEM model was examined in more detail in Tao et al. (2007).

\section{Wave forces}

The dimensionless wave forces on the interior cylinder and exterior porous cylinder due to a plane wave vs. $a / b$ for $G_{0}=1.0$ are plotted in Fig. 7. Again, in order to compare with the analytical solutions of Wang and Ren (1994), the SBFEM results shown in the figure are calculated using the same parameter values as in Wang and Ren (1994). It can be seen that the SBFEM results calculated with only 4 surface elements on the cylinder boundary are almost identical to 
the analytical solutions. The essential feature that the wave force on the exterior porous cylinder reduces to zero near $a / b=0.2$ for $C_{w}=0.4$ is reproduced extremely well by the present SBFEM model. In contrast to the wave run-up calculations, where more surface elements are required as $k a$ and $k b$ increase, it is observed from Fig. 7, that the convergence of the SBFEM model for the wave force computation is at best weakly dependent on the dimensionless parameter $k a$ and $k b$.

Fig. 8 shows the variation of the total force $|2 \pi R P|$ with the short-crestedness (i.e., $k_{y} / k_{x}$ ) of the waves. The SBFEM results on wave forces shown in Fig. 8 were calculated either on the interior cylinder with the porosity of the exterior cylinder $G_{0}=+\infty$ and $a=1 \mathrm{~m}$, or on the exterior cylinder with $G_{0}=0$ and $b=1 \mathrm{~m}$. As can be seen in the figure, the SBFEM model gave almost identical solutions to the analytical solutions of Zhu (1993) with merely 6 surface elements discretized on the cylinder boundary. Note that the total force decreases rapidly as the incident wave becomes more and more short-crested (i.e., higher $k_{y} / k_{x}$ values).

\section{PARAMETRIC STUDY}

\section{Wave forces}

\section{Influence of wave parameters}

Zhu (1993) calculated the effective inertia coefficient $C_{M}$ and the linear drag coefficient $C_{D}$ per unit height of a single solid cylinder, and the results were plotted against the variation of the ratio $k_{y} / k_{x}$. Four cases were calculated coincidental with the same $k_{x} a$ to show that $C_{M}$ and $C_{D}$ are invariants for a fixed $k a$. In order to examine whether this characteristic is present in a concentric porous cylindrical structure as well, four cases similar to Zhu (1993) are chosen: 1) $k_{x}=0.4 \mathrm{~m}^{-1}$, $a=2.5 \mathrm{~m}$;2) $k_{x}=0.5 \mathrm{~m}^{-1}, a=2.0 \mathrm{~m}$;3) $k_{x}=0.8 \mathrm{~m}^{-1}, a=1.25 \mathrm{~m}$; and 4) $k_{x}=1.0 \mathrm{~m}^{-1}$, $a=1.0 \mathrm{~m}$. In the present calculation, the ratio $b / a$ is fixed at 2 , thus giving $k_{x} b=2$ and $k_{x} a=1$ for all the above four cases with porosity $G_{0}=1$. Two extreme cases for $G_{0}=+\infty$ (inner cylinder only) and $a=0$ (outer cylinder only) are also chosen for comparison. From Figs. 9 and 10, it is clearly seen that $C_{M}$ and $C_{D}$ are still invariants of $k a$ and $k b$. Eq. (86) indicates that the total horizontal force is proportional to $k_{x}$ while $k, a$ and $b$ are fixed. Thus the largest wave induced 
forces for short-crested waves are smaller than those induced by plane waves with the same total wave number because of the standing wave component.

The variations of the total horizontal forces $|2 \pi R P|$ on the interior and exterior cylinders by plane incident waves $v s$. $k a$ are shown in Fig. 11 for $a=1 \mathrm{~m}, b / a=2$ and $G_{0}=1$. It can be seen that the total horizontal forces fluctuate and peak attenuation is observed. It is interesting to note that as the horizontal force on the interior cylinder (solid line) reaches a peak, the force on the exterior cylinder (dashed line) reaches a trough. One the other hand, however, when the horizontal force on the interior cylinder reaches a trough, the force on the exterior cylinder does not correspondingly show a peak. The reduction in the amplitude of wave forces on the interior cylinder in the presence of the exterior porous cylinder is clearly evident when compared to the corresponding wave forces on the single impermeable cylinder (denoted by the dotted line in Fig. $11)$.

The variations of the total horizontal forces $(|2 \pi R P|)$ on the interior and exterior cylinders with $G_{0}=1 v s$. the ratio $k_{y} / k_{x}$ for different $k_{x} a$ values ( $k_{x}$ from 0.2 to $1.2 \mathrm{~m}^{-1}$ ) with $a=1 \mathrm{~m}, b=2 a$ are shown in Figs. 12 and 13 respectively. They show similar trends of peaks and troughs found in Fig. 11. These variations of maximum wave forces on the interior and exterior cylinders provide a means of minimizing wave loads on both cylinders.

Three types of variation patterns in wave forces are observed in Fig. 12. For small $k_{x} a\left(k_{x} a=\right.$ 0.2 ), the horizontal force increases gently to a peak and then decreases mildly. For moderate $k_{x} a\left(k_{x} a=0.4,0.6\right)$, the curves are seen to slowly translate leftwards (starting with larger $\left.k a\right)$. Initially, the horizontal force is seen descending slightly, followed by a sharp drop off and finally becoming nearly flat. For large $k_{x} a\left(k_{x} a=0.8,1.0,1.2\right)$, the curve translates further leftwards. All forces show a trend of dramatic dip to almost steady small values at large values of $k_{y} / k_{x}$. In Fig. 13 , there are troughs near zero at each $k_{x} a$ indicating extremely low wave forces on the exterior cylinder. This important characteristic in the forces can be effectively applied in a design to reduce the wave impact on coastal and offshore porous structures. 


\section{Influence of system configuration}

The effect of the annular spacing between the cylinders is investigated and the results are presented in terms of the total force variation on the ratio $a / b$ in Figs. 14 and 15, where $b$ is fixed at $b=10 \mathrm{~m}$. The wave number in $x$ direction is taken as $k_{x}=0.1 \mathrm{~m}^{-1}$ and the ratio $k_{y} / k_{x}$ is ranged from 0 to 5 .

Fig. 14 shows lower wave force on the interior cylinder with smaller radius $a$ for a fixed radius, $b$, of the exterior cylinder. Also, the total horizontal force on the interior cylinder increases monotonically as waves approach plane waves and fluctuates as the incident waves become more short-crested. The variations, shown in Fig. 15 of the total horizontal forces on the exterior cylinder $(|2 \pi b P|)$ are quite different for different $k_{y} / k_{x}$ values and no common trends can be found. Fig. 15 also shows that the total horizontal forces on the exterior cylinder resulted from shortcrested waves fluctuate in values with $a / b$ whereas a trend of monotonically decreasing with $a / b$ is clearly observed for plane incident waves. Generally, less short-crestedness results in larger wave forces on external cylinder except in the neighborhood of troughs. These findings are considered important for the designer in order to minimize the hydrodynamic loads on practical structures.

The influence of the porous-effect parameter $G_{0}$ on wave forces is examined in Fig. 16 with diameter ratio of $b / a=2$ and $a=1 \mathrm{~m}$. Three cases are presented in the figure: (1) $k_{x} a=k_{y} a=1$, $k a=\sqrt{2} ;(2) k_{x} a=\sqrt{2}, k_{y} a=0, k a=\sqrt{2} ;$ and (3) $k_{x} a=1, k_{y} a=0, k a=1$, corresponding to a short-crested incident wave and two plane incident waves, respectively. It can be seen that the total horizontal force on the interior cylinder increases monotonically, while the total horizontal force of the exterior cylinder decreases monotonically as $G_{0}$ increases. The variation in forces is slow at higher $G_{0}$, approaching an asymptotic value in each case.

\section{Surface elevation}

It is interesting to study the changes in the wave surface elevation in the vicinity of the concentric porous cylindrical structure for varying incident wave parameters and structure configuration. Fig. 17 shows the resulting wave amplitude and corresponding phase contours resulting from plane, short-crested, and standing incident wave interaction with the structure for configuration 
parameters: $G_{0}=1.0, a=1 \mathrm{~m}$ and $b=2 \mathrm{~m}$. The amplitudes shown in Fig. 17, are nondimensionalized as $|\eta| / A$ ( $\eta$ and $A$ represent free surface elevation and the amplitude of incident wave respectively), and the phase values plotted in Fig. 17 are in the range of $[-\pi \sim+\pi]$.

It is clear that the diffracted wave patterns of short-crested waves are more complicated than those of plane waves. As one will expect, all the equi-amplitude plots are symmetric with respect to the longitudinal $(x)$ axis leading to zero force in transverse $(y)$ direction. Moreover, the diffracted wave pattern resulting from a standing wave shown in Fig. 17 is symmetric in both $x$ and $y$ planes, generating zero horizontal force in both longitudinal and transverse directions. It is seen that the amplitude of the diffracted short-waves in the lee region is smaller than that of a plane wave, and the region for the large amplitude waves in front of the cylinders resulting from short-crested waves is also smaller than its plane wave counterpart. Further calculations on different wave number ratio $k_{y} / k_{x}$ revealed that such tendencies are more pronounced as the incident waves become more short-crested.

The thick lines in phase contours represent changes from $\pi$ to $-\pi$. The amphidromic points, where equi-phase lines converge and the wave amplitude vanishes, are seen clearly formed for short-crested incident waves (see Fig. 17). Similar to the feature observed by Zhu (1993) for short-crested wave diffraction by a single impermeable cylinder, the phases near two adjacent amphidromic points rotate from $-\pi$ to $+\pi$ clockwise and counter-clockwise around the amphidromic points respectively in the region surrounding the structure. Further calculations on different wave number ratio $k_{y} / k_{x}$ revealed that the density of the amphidromic points increases as the wave crests become shorter. It is interesting to note that the equi-phase lines for the short-crested waves become almost parallel to each other in the region downstream, where no obvious amphidromic points formed, indicating that the waves in the lee side of the structure are no longer short-crested. As one would expect for the standing incident wave component, the amplitude and phase contours maintain symmetry in the $x$ - and $y$-plane. The amplitudes in the transverse directions are small compared to their inline values, with a faster variation in the corresponding phase contours.

It is worth stressing that all the numerical results presented in this paper are calculated using 
the SBFEM model with no more than 8 surface elements discretized over the cylinder boundary. The only exceptions are the results in Figs. 12 and 13, where 16 surface elements are used due to larger values of $k a$ and $k b$, so that the variation of the total horizontal force with varying wave number ratio $k_{y} / k_{x}$ becomes clear.

The computational times (recorded on a $2 \mathrm{GHz}$ Pentium IV PC and MATLAB 7.1) of the SBFEM solutions for all the cases presented here are less than $3 \mathrm{sec}$. Thus, it clearly demonstrates the superiority of the present method in significantly outperforming its counterparts in currently available finite-element or boundary-element methods for similar problems. Such computational efficiency and accuracy ensure a great potential of direct application of the present method to many engineering problems, especially in ocean engineering.

The present SBFEM approach shown in this paper is valid for wave diffraction by a porous circular cylinder system of bounded and unbounded domains. However, by means of domain decomposition, and choosing appropriate forms of the radial function $a(\xi)$ and scaling centers for different sub-domains, the authors have recently extended the SBFEM to solve other geometries while maintaining dominant features of the present SBFEM (Song and Tao, 2008).

\section{CONCLUSIONS}

The versatility of the newly developed semi-analytical scaled boundary finite-element method is demonstrated in this paper in considering the interaction of short-crested waves with a concentric porous cylindrical structure. In contrast to the conventional boundary-element method, which has been widely applied to wave-structure interaction problem in unbounded domain, the SBFEM model developed for concentric porous cylinders requires no assistance from any fundamental solutions. A unique advantage of this method is that a reduction of one in the spatial dimensions is achieved. Thus only the body boundary is discretized with surface finite-elements. Excellent computational efficiency and accuracy of the SBFEM model has been demonstrated, as the governing equations are solved analytically in the radial direction.

Solutions for the interaction of plane and short-crested waves with a concentric porous cylindrical structure are achieved. The present numerical method is shown to reproduce the results of 
available analytical solutions for all the physical properties including wave run-up and wave forces very accurately and at very low computational cost. Detailed parametric study revealed that the trends of wave forces and surface elevation patterns for short-crested waves are much more complex than the ones for the plane incident waves. General trends of wave force on the ratio $a / b$ are discussed with potential to minimize the wave impact in a particular design. The results presented here should be found useful in the design of coastal and ocean structures.

The present SBFEM model is demonstrated to be very efficient yielding accurate results in the wave force and run-up calculations with no more than 8 elements for small and moderate $k a$ and $k b$ values. Thus the model is believed to possess a significant advantage in its application to more complicated coastal and offshore structures geometry.

\section{REFERENCES}

Chwang, A.T. (1983). “A porous wavemaker theory.” J. Fluid Mech., 132, 395-406.

Chwang, A.T., and Chan, A.T. (1998). "Interaction between porous media and wave motion." Annu. Rev. Fluid Mech., 30, 53-84.

Chwang, A.T., and Dong, Z. (1984). "Wave-trapping due to a porous plate." Proc. 15th Symp. on Naval Hydrodynamics, Hamburg, Germany, 407-417.

Chwang, A.T., and Li, W. (1983). "A piston-type porous wavemaker theory.” J. Eng. Math., 17, 301-313.

Chwang, A.T., and Wu, J. (1994). "Wave scattering by submerged porous disk." J. Engrg. Mech., 120(12), 2575-2587.

Dalrymple, R.A., Losada, M.A., and Martin, P.A. (1991). "Reflection and transmission from porous structures under oblique wave attack." J. Fluid Mech., 224, 625-644.

Darwiche, M.K.M., Williams, A.N., and Wang, K.-H. (1994). "Wave interaction with semiporous cylindrical breakwater." J. Waterway, Port, Coastal, Ocean Eng., 120(4), 382-403. 
Deeks, A.J., and Cheng L. (2003). "Potential flow around obstacles using the scaled boundary finite-element method.” Int. J. Numer. Meth. Fluids, 41(7), 721-741.

Faltas, M.S. (1996). “On oblique waves forcing by a porous cylindrical wall.” Internat. J. Math. \& Math. Sci., 19(2), 351-362.

Fuchs, R.A. (1952). "On the theory of short-crested oscillatory waves.” Gravity Waves, National Bureau of Standards Circular No. 521, Department of Commerce, USA, 187-200.

Fuhrman, D.R., and Madsen, P.A. (2006). "Short-crested waves in deep water: a numerical investigation of recent laboratory experiments.” J. Fluid Mech., 559, 391-411.

Guiney, D.C., Noye, B.J., and Tuck E.O. (1972). "Transmission of water waves through small apertures.” J. Fluid Mech., 55, 149-161.

Huang, L.H., and Chao, H.I. (1992). "Reflection and transmission of water wave by porous breakwater." J. Waterway, Port, Coastal, Ocean Eng., 118(5), 437-452.

Li, B., Cheng, L., and Deeks, A.J. (2004). "Wave diffraction by vertical cylinder using the scaled boundary finite element method." WCCM VI \& APCOM'04, Beijing, China.

Li, Y., Sun, L., and Teng, B. (2003). "Wave action on double-cylinder structure with perforated outer wall." Proc. 22nd Int. OMAE Conf., CD-ROM: OMAE2003-37094.

Madsen, O.S. (1974). "Wave transmission through porous structures." J. Wtrwy., Harb. and Coast. Engrg. Div., 100(3), 169-188.

Morse P.M., and Feshbach H. (1953). Methods of Theoretical Physics. McGraw-Hall Book Comany, New York, USA.

Nasser, M.S., and McCorquodale J.A. 1974. "Experimental Study of Wave Transmission.” J. Wtrwy., Harb. and Coast. Engrg. Div., 100(4), 279-286. 
Porter, D. (1972). “The transmission of surface waves through a gap in a vertical barrier." Proc. Camb. Phil. Soc., 71, 411-421.

Song, H., and Tao, L. (2008). "Scaled boundary FEM solution of wave diffraction by a square caisson.” Proc. 27th Int. OMAE Conf., Paper No. OMAE2007-57279.

Song, Ch., and Wolf, J.P. (1997). “The scaled boundary finite-element method - alias consistent infinitesimal finite-element cell method - for elastodynamics." Comput. Method Appl. M., 147, 329-355.

Tao, L., Song, H., and Chakrabarti, S. K. (2007). "Scaled boundary FEM solution of short-crested wave diffraction by a vertical cylinder." Comput. Method Appl. M., 197, 232-242.

Taylor, G. (1956). "Fluid flow in regions bounded by porous surfaces." Proc. R. Soc. Lond. A, 234(1199), 456-475.

Tsai, C.P., Jeng, D.S., and Hsu, J.R.C. (1994). “Computations of the almost highest short-crested waves in deep water." Appl. Ocean Res., 16(6), 317-326.

Tuck, E.O. (1971). "Transmission of water waves through small apertures.” J. Fluid Mech., 49, $65-74$

Vijayalakshmi, K, Neelamani, S., Sundaravadivelu, R., and Murali, K. (2007). "Wave runup on a concentric twin perforated circular cylinder." Ocean Eng., 34(2), 327-336.

Wang, K.-H., and Ren, X. (1993). "Water waves on flexible and porous breakwater." J. Engrg Mech., 119(5), 1025-1047.

Wang, K.-H., and Ren, X. (1994). "Wave interaction with a concentric porous cylinder system." Ocean Eng., 21(4), 343-360.

Williams, A.N., and Li, W. (1998). "Wave interaction with a semi-porous cylindrical breakwater mounted on a storage tank." Ocean Eng., 25(2-3), 195-219. 
Wolf, J.P. (2003). The scaled boundary finite element method. John Wiley \& Sons Ltd, Chichester, England.

Yu, X., and Chwang, A.T. (1994a). "Wave-induced oscillation in harbor with porous breakwaters." J. Waterway, Port, Coastal, Ocean Eng., 120(2), 125-144.

Yu, X., and Chwang, A.T. (1994b). "Wave motion through porous structures." J. Engrg. Mech., 120(5), 989-1008.

Yu, X., and Chwang, A.T. (1994c). "Water waves above submerged porous plate.” J. Engrg. Mech., 120(6), 1270-1282.

Zhong, Z., and Wang, K.H. (2006). "Solitary wave interaction with a concentric porous cylinder system.” Ocean Eng., 33(7), 927-949.

Zhu, S. (1993). "Diffraction of short-crested waves around a circular cylinder." Ocean Eng., 20(4), $389-407$.

Zhu, S., and Moule, G. (1994). "Numerical calculation of forces induced by short-crested waves on a vertical cylinder of arbitrary cross-section.” Ocean Eng., 21(7), 645-662. 


\section{List of Figures}

1 Ekofisk Gravity Structure (Courtesy ConocoPhillps). . . . . . . . . . . . . . . 32

2 Definition sketch of wave interaction with a concentric porous cylindrical structure. 33

3 The coordinate definition of SBFEM. . . . . . . . . . . . . . . . 34

$4 \quad$ Scaled boundary finite element mesh for a concentric porous cylindrical structure. . 35

5 Run-up of an incident plane wave around the interior and exterior cylinders. . . . . 36

6 Run-up of a short-crested wave on the interior cylinder at $a=1 \mathrm{~m}$ and the exterior cylinder at $b=2 \mathrm{~m} \ldots \ldots \ldots \ldots \ldots \ldots \ldots \ldots \ldots \ldots$

$7 \quad$ Wave force due to a plane wave on the interior and exterior cylinders for $C_{w}=0.4 . \quad 38$

8 Total wave force $|2 \pi R P|$ due to short-crested waves on a single solid cylinder at

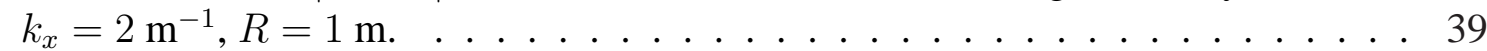

9 Variation of the effective inertial coefficient $C_{M}$ vs. the ratio $k_{y} / k_{x}$ at $k_{x} a=1$,

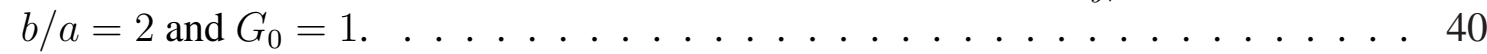

10 Variation of the effective drag coefficient $C_{D} v s$. the ratio $k_{y} / k_{x}$ at $k_{x} a=1, b / a=$ 2 and $G_{0}=1 \ldots \ldots \ldots \ldots \ldots \ldots \ldots \ldots \ldots \ldots \ldots$

11 Variation of the total horizontal forces on the interior and exterior cylinder by plane incident wave $v s . k a$ at $a=1 \mathrm{~m}, b / a=2$ and $G_{0}=1 \ldots \ldots \ldots \ldots$. . . . 42

12 Variation of the total horizontal force on the interior cylinder $v s$. the ratio $k_{y} / k_{x}$ at $a=1 \mathrm{~m}, b / a=2$ and $G_{0}=1 \ldots \ldots \ldots \ldots \ldots \ldots \ldots$

13 Variation of the total horizontal force on the exterior cylinder $v s$. the ratio $k_{y} / k_{x}$ at $a=1 \mathrm{~m}, b / a=2$ and $G_{0}=1 \ldots \ldots \ldots \ldots \ldots \ldots \ldots$

14 Variation of the total horizontal force on the interior cylinder $v s$. the ratio $a / b$ at different $k_{y}\left(b=10 \mathrm{~m}, k_{x}=0.1 \mathrm{~m}^{-1}\right) \ldots \ldots \ldots \ldots \ldots \ldots \ldots$

15 Variation of the total horizontal force on the exterior cylinder $v s$. the ratio $a / b$ at different $k_{y}\left(b=10 \mathrm{~m}, k_{x}=0.1 \mathrm{~m}^{-1}\right) \ldots \ldots \ldots \ldots \ldots \ldots \ldots \ldots$

16 Variations of the total horizontal forces of the cylinders $v s$. the porous-effect parameter $G_{0}(b / a=2$ and $a=1 \mathrm{~m}) \ldots \ldots \ldots \ldots \ldots \ldots$. . . . . . . . . . . .

17 Equi-amplitude contours (left) and Equi-phase contours (right) for the incident plane, short-crested, and standing waves, respectively. . . . . . . . . . . . . . 48 


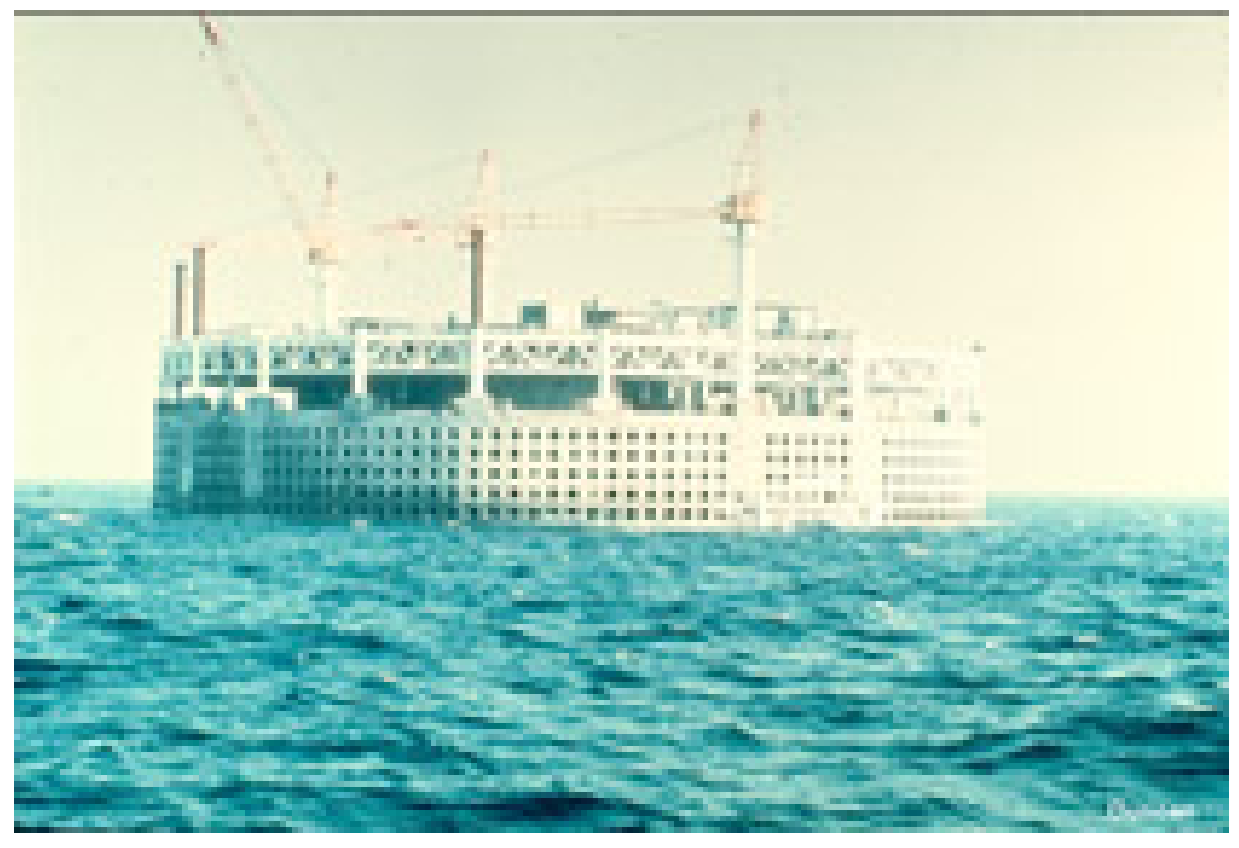

FIG. 1. Ekofisk Gravity Structure (Courtesy ConocoPhillps). 

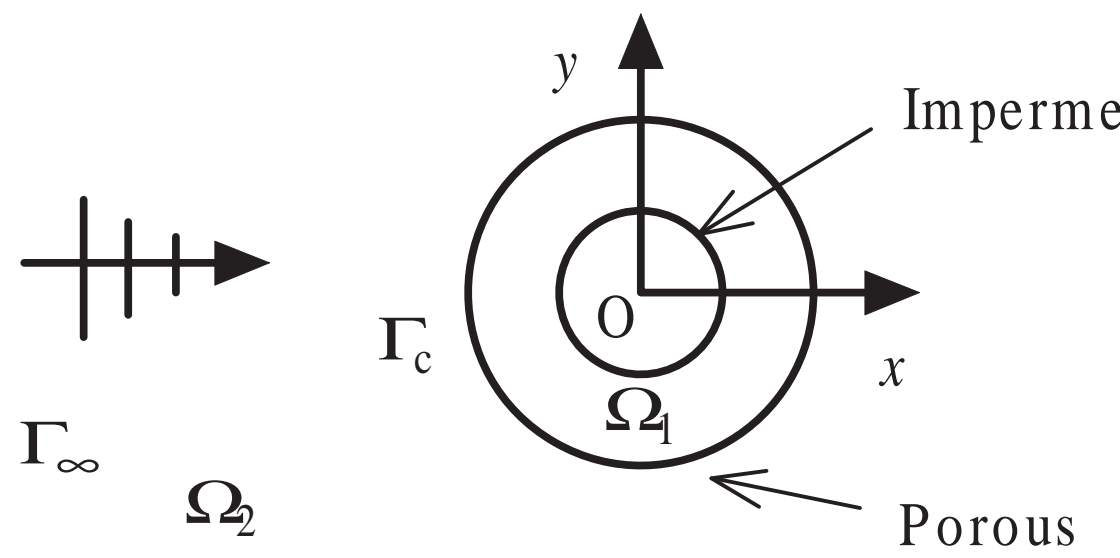

$\Gamma_{\infty}$

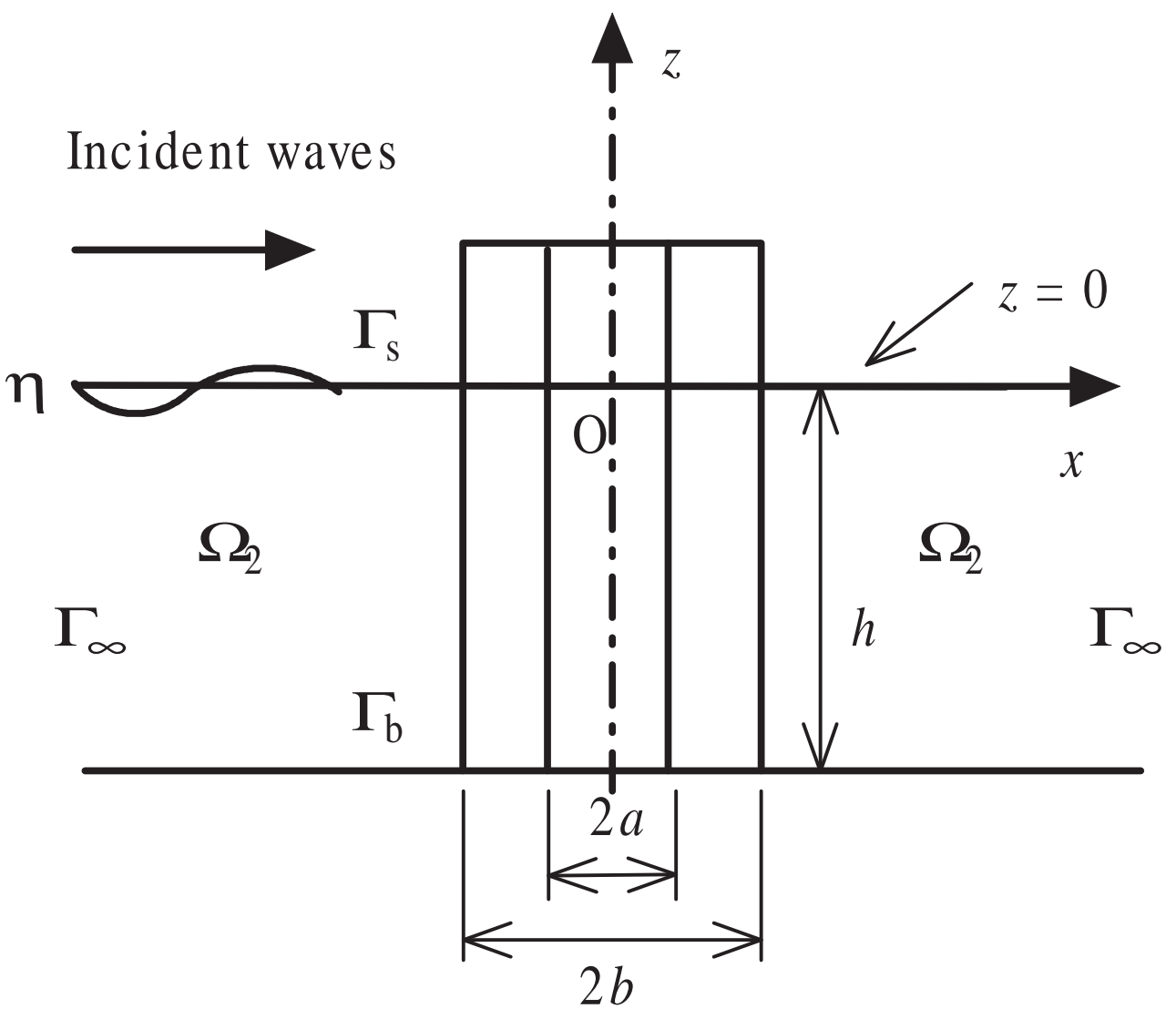

FIG. 2. Definition sketch of wave interaction with a concentric porous cylindrical structure. 


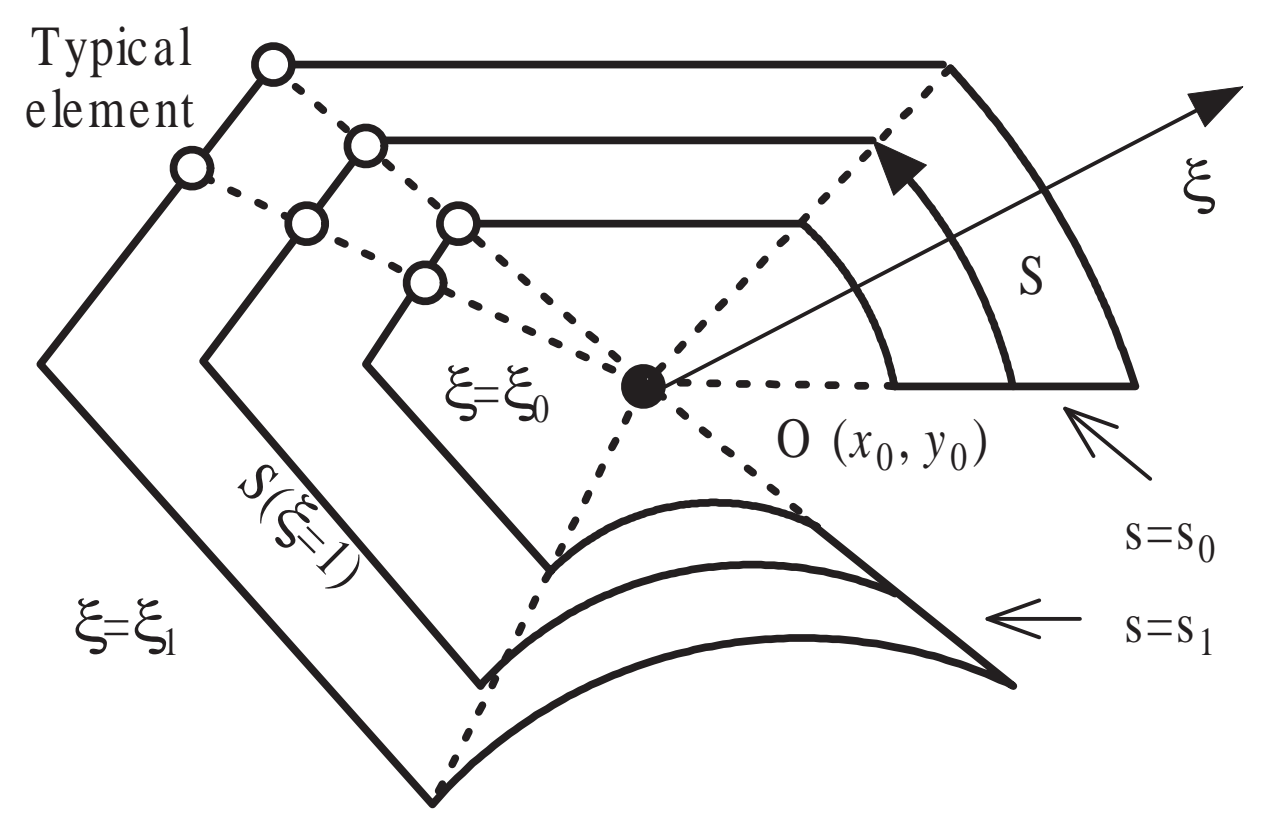

FIG. 3. The coordinate definition of SBFEM. 


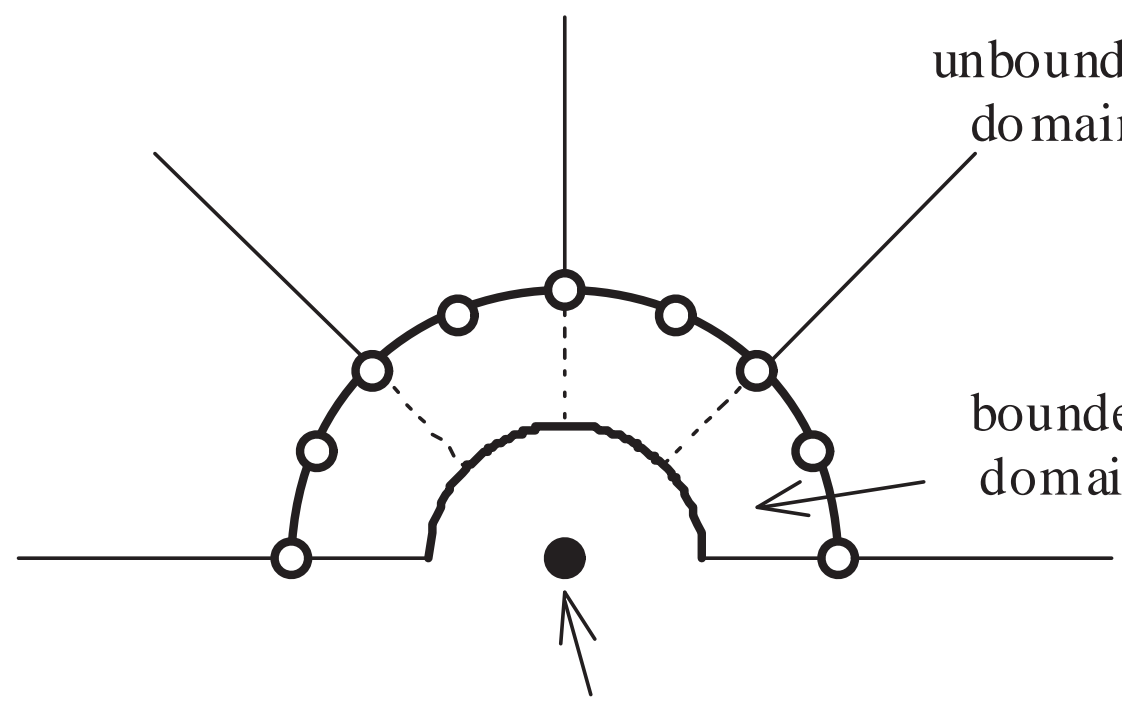

Scalgoente

FIG. 4. Scaled boundary finite element mesh for a concentric porous cylindrical structure. 


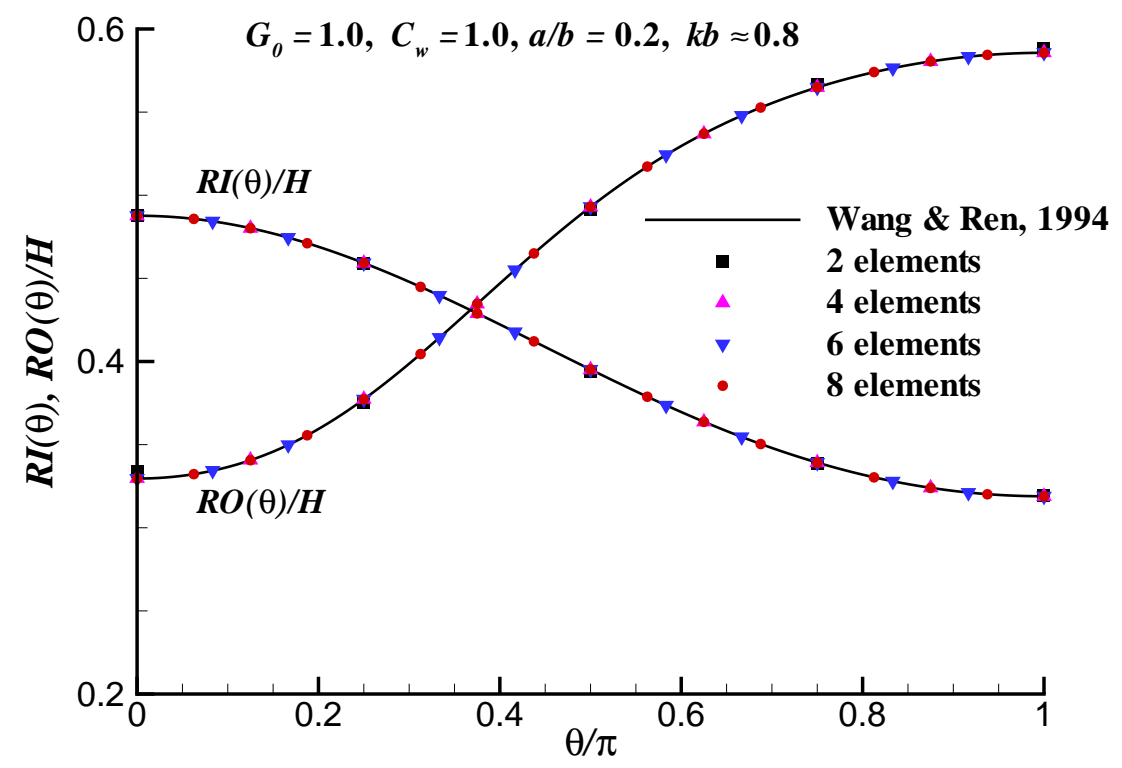

FIG. 5. Run-up of an incident plane wave around the interior and exterior cylinders. 


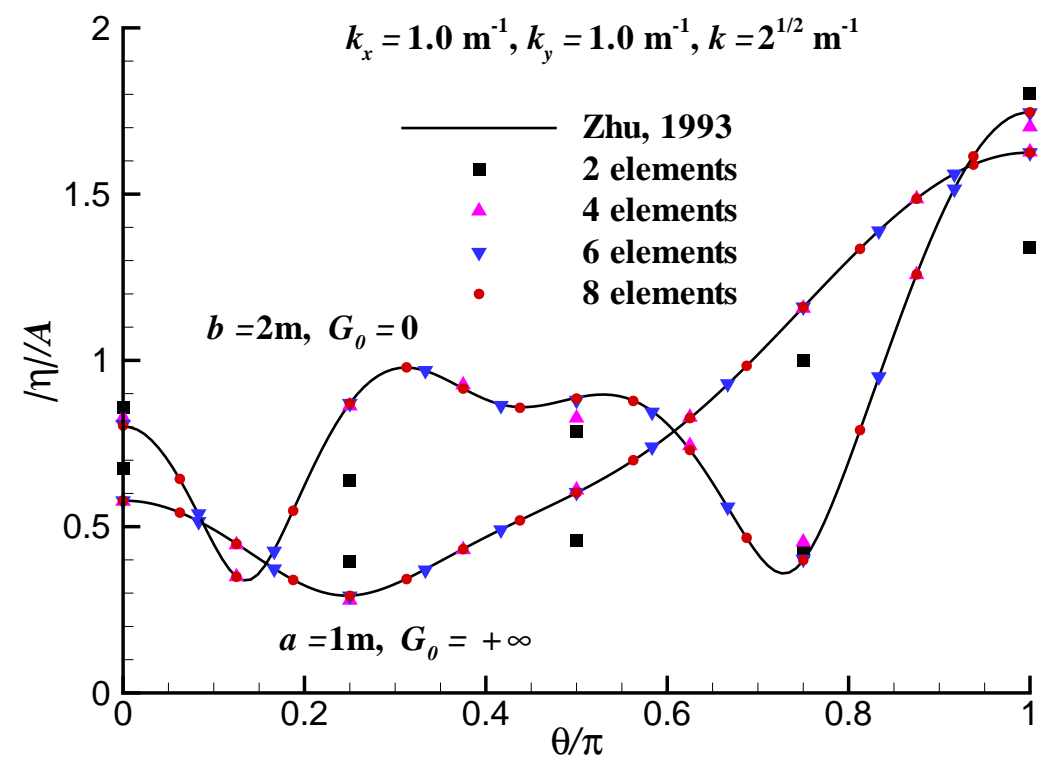

FIG. 6. Run-up of a short-crested wave on the interior cylinder at $a=1 \mathbf{m}$ and the exterior cylinder at $b=2 \mathbf{m}$. 


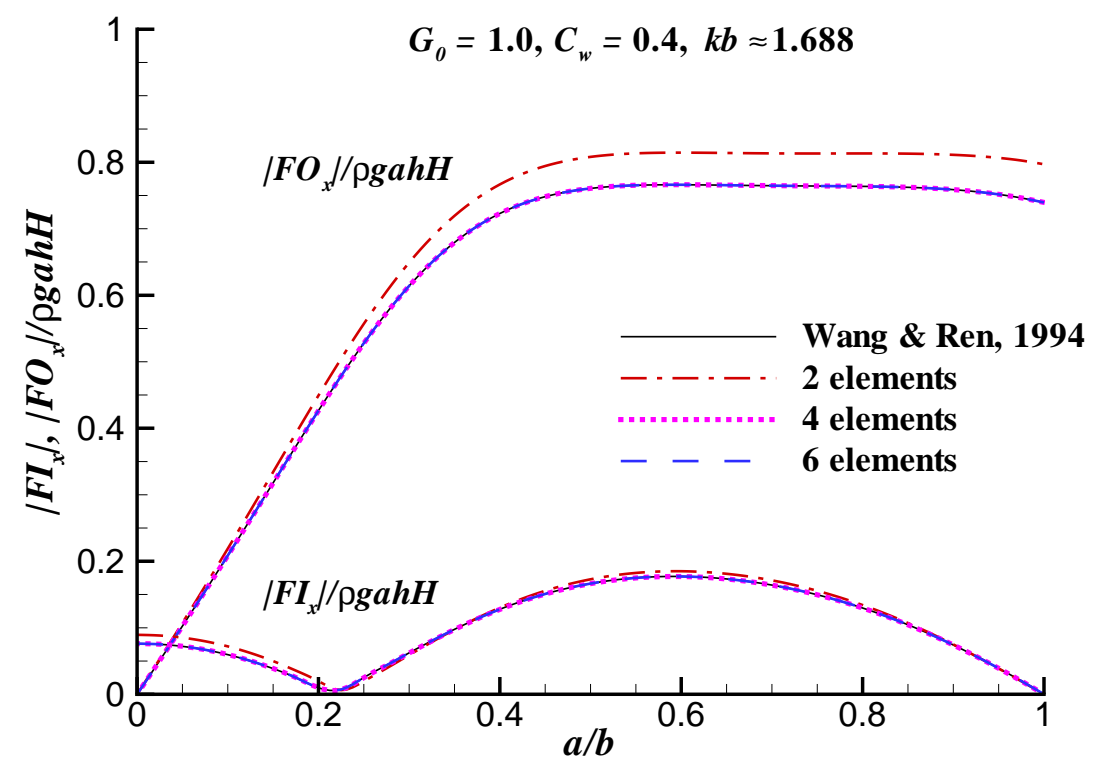

FIG. 7. Wave force due to a plane wave on the interior and exterior cylinders for $C_{w}=0.4$. 


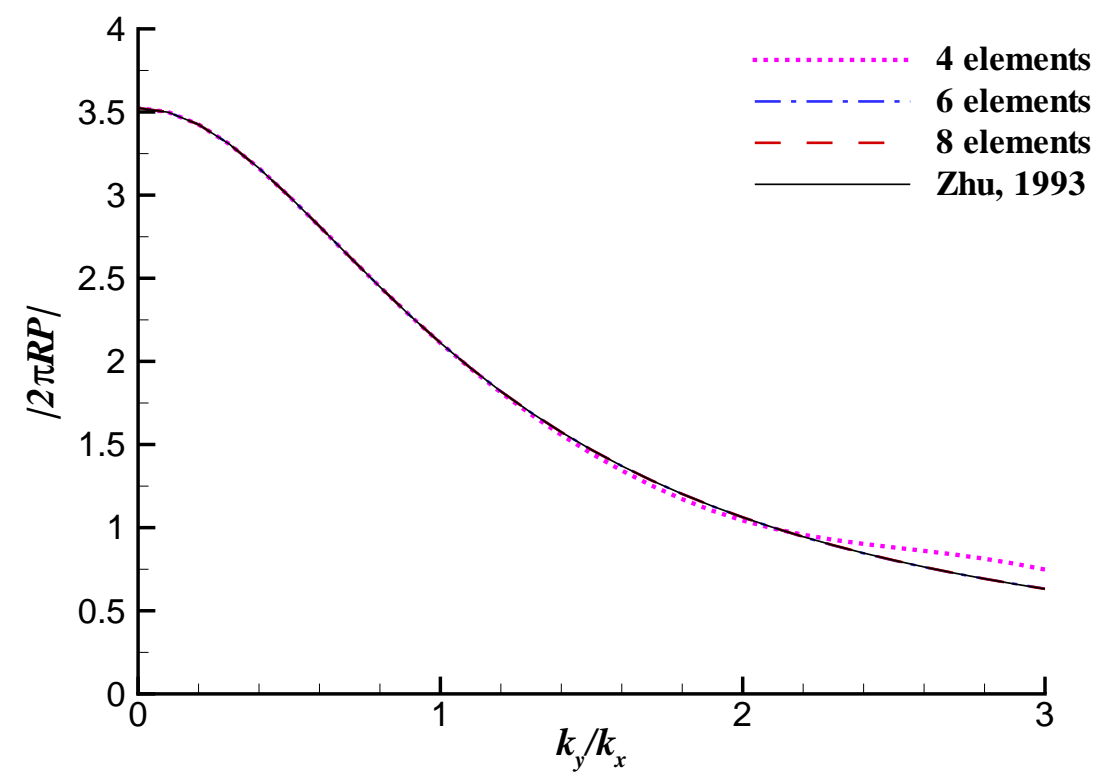

FIG. 8. Total wave force $|2 \pi R P|$ due to short-crested waves on a single solid cylinder at $k_{x}=2 \mathbf{m}^{-1}, R=1 \mathbf{m}$. 


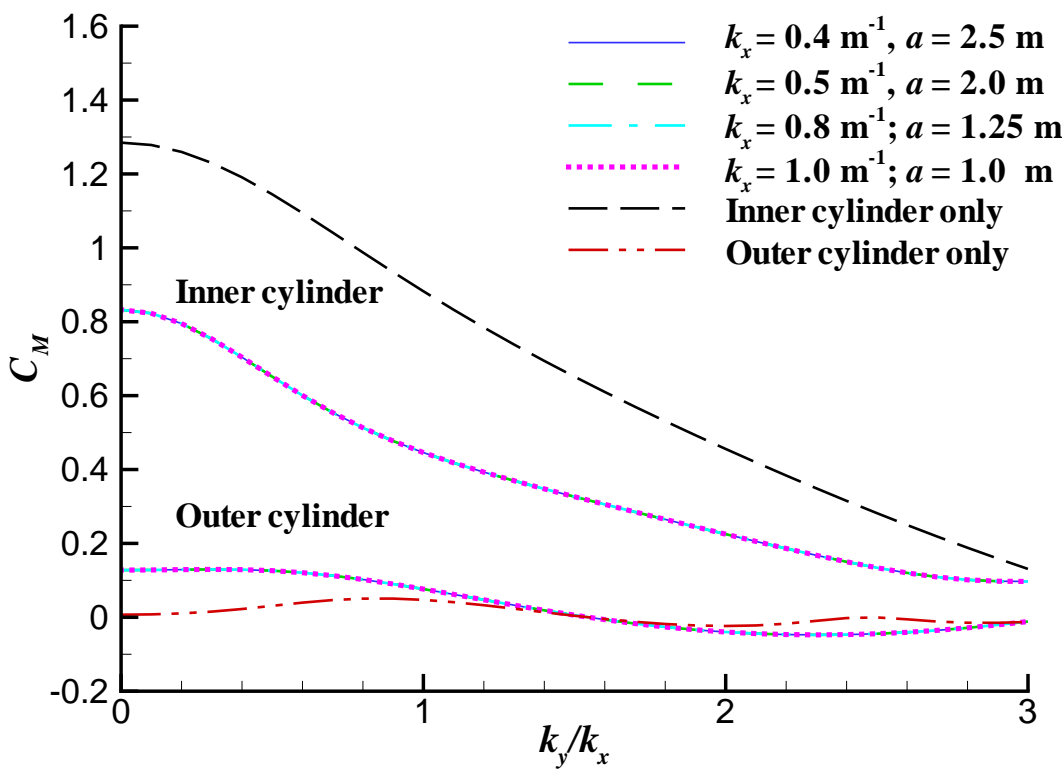

FIG. 9. Variation of the effective inertial coefficient $C_{M} v s$. the ratio $k_{y} / k_{x}$ at $k_{x} a=1$, $b / a=2$ and $G_{0}=1$. 


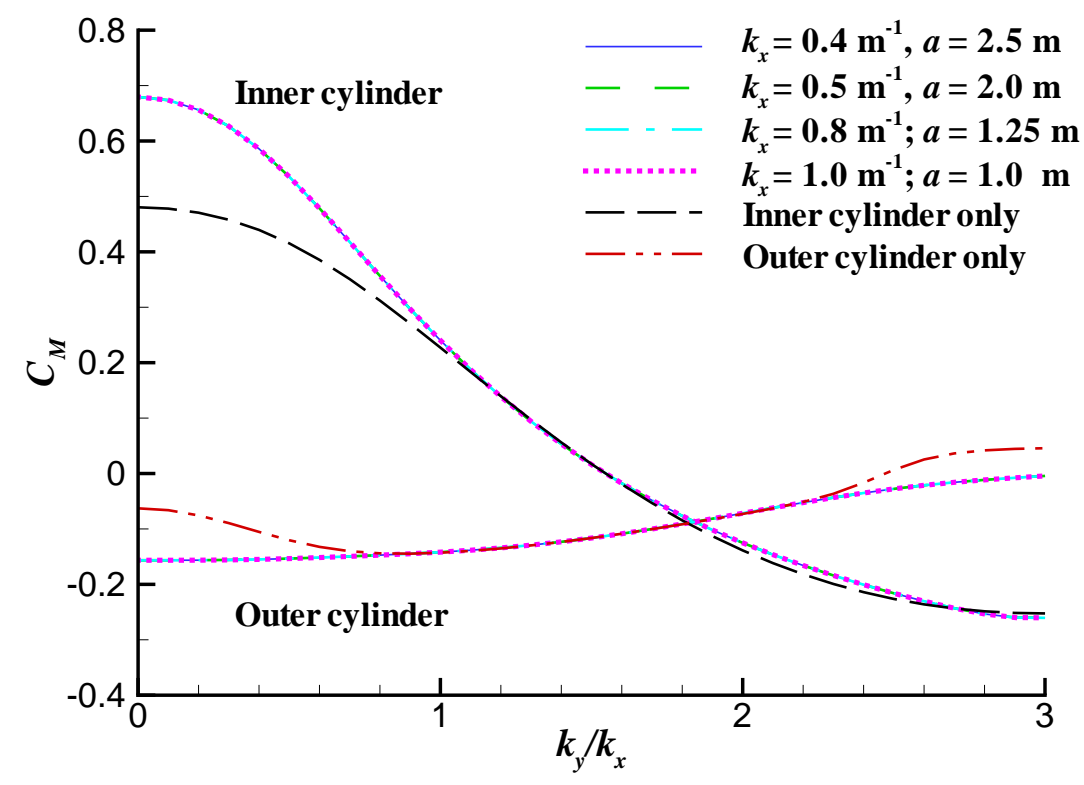

FIG. 10. Variation of the effective drag coefficient $C_{D} v s$. the ratio $k_{y} / k_{x}$ at $k_{x} a=1$, $b / a=2$ and $G_{0}=1$. 


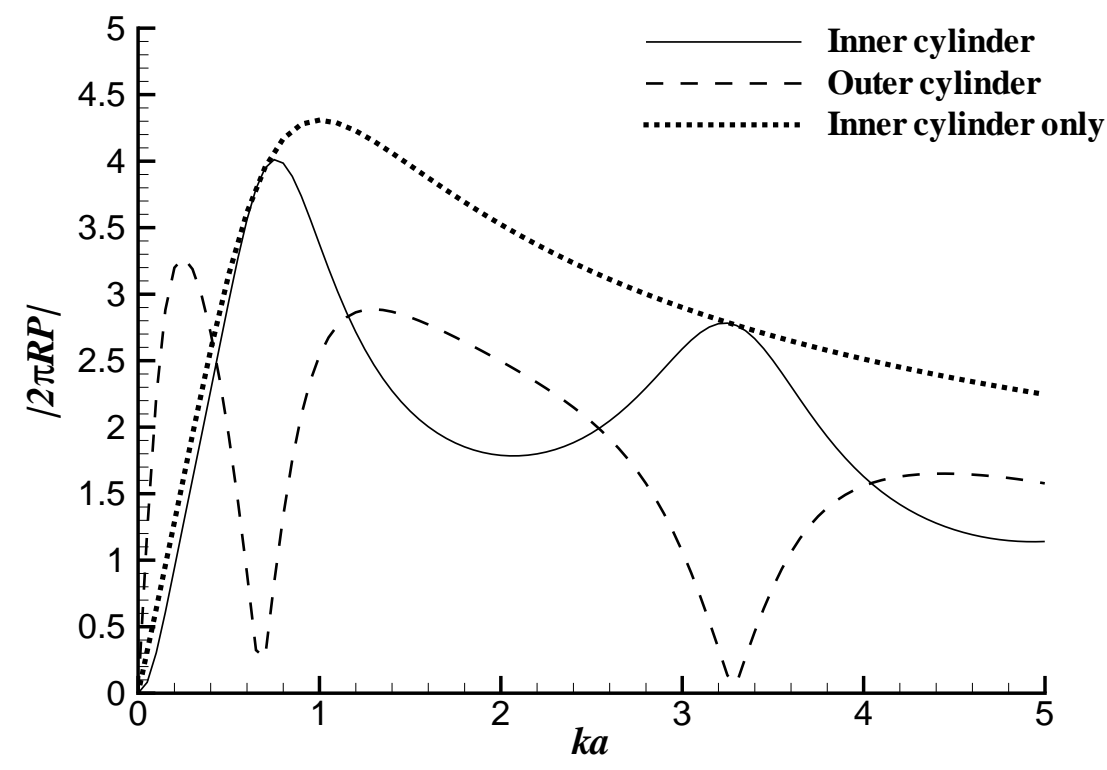

FIG. 11. Variation of the total horizontal forces on the interior and exterior cylinder by plane incident wave $v s . k a$ at $a=1 \mathbf{m}, b / a=2$ and $G_{0}=1$. 


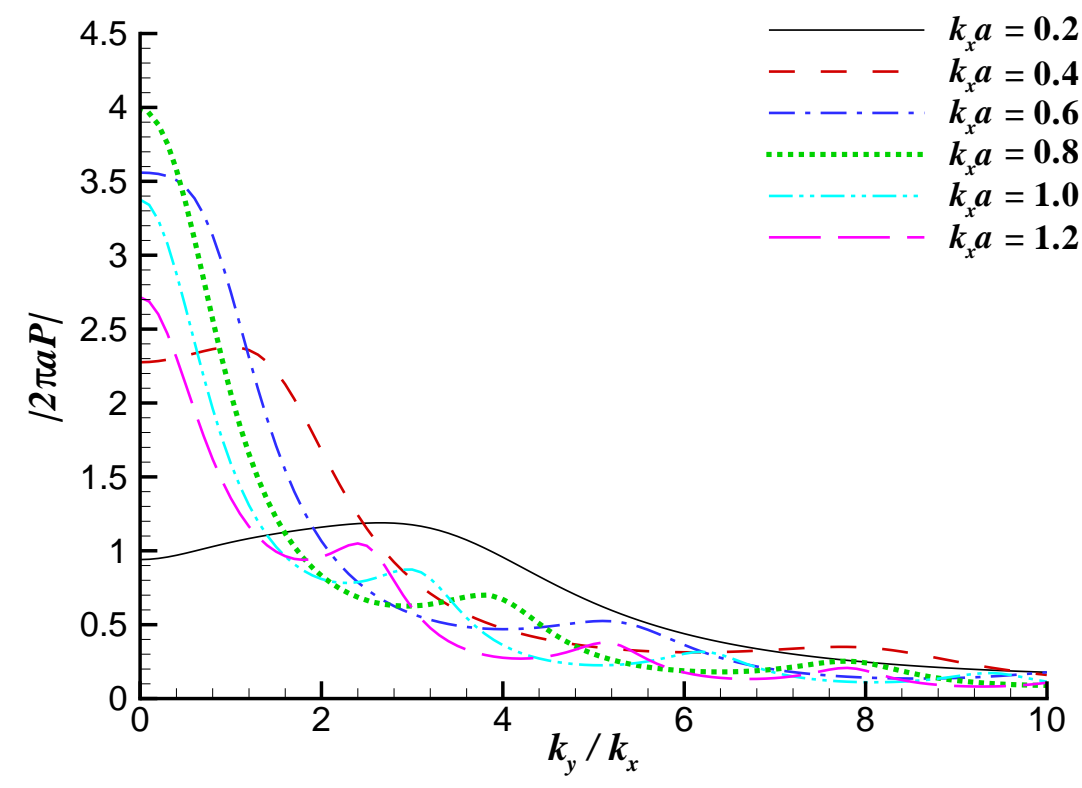

FIG. 12. Variation of the total horizontal force on the interior cylinder $v s$. the ratio $k_{y} / k_{x}$ at $a=1 \mathbf{m}, b / a=2$ and $G_{0}=1$. 


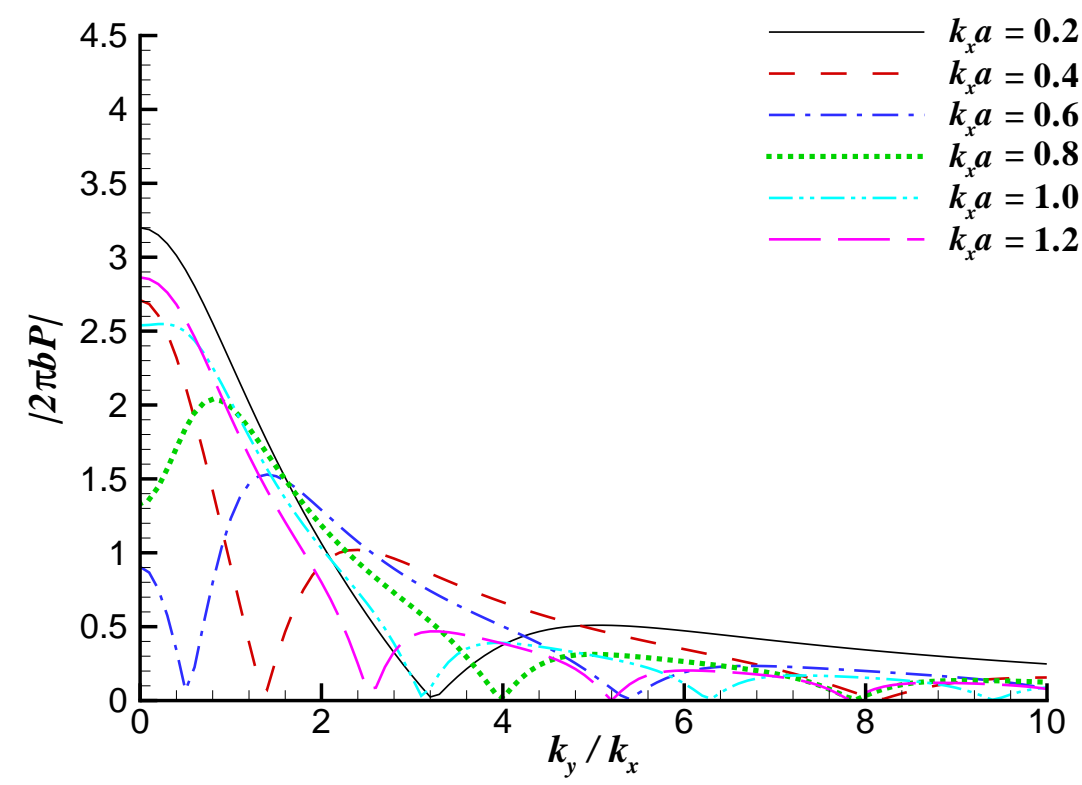

FIG. 13. Variation of the total horizontal force on the exterior cylinder $v s$. the ratio $k_{y} / k_{x}$ at $a=1 \mathbf{m}, b / a=2$ and $G_{0}=1$. 


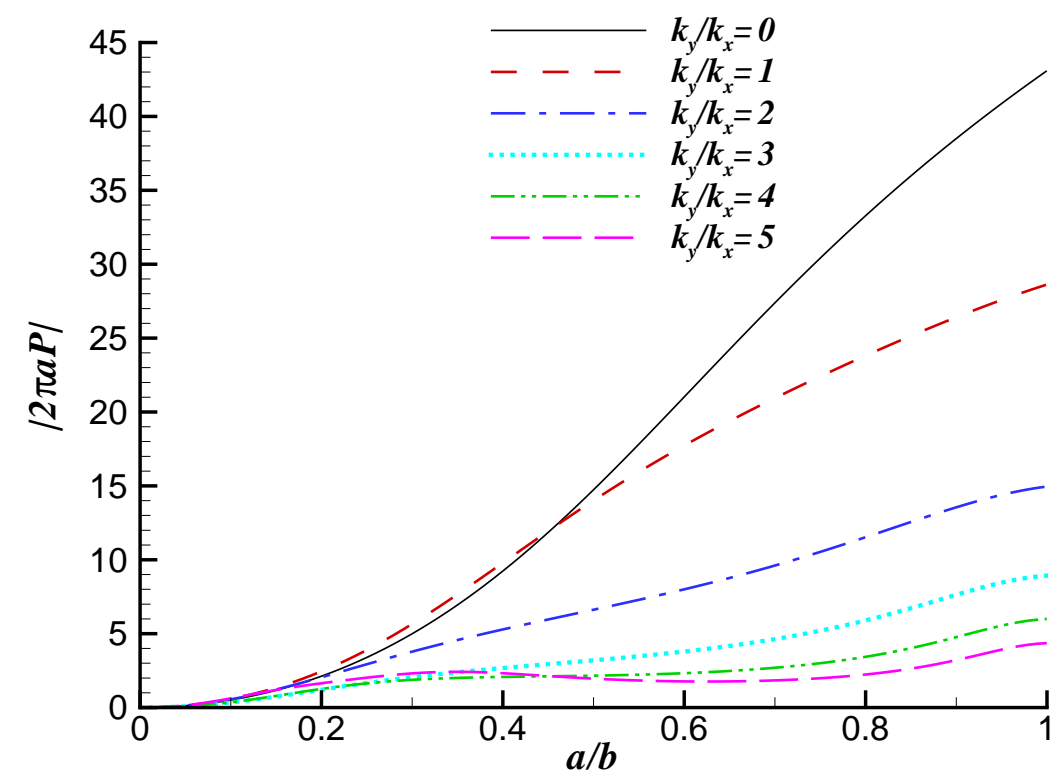

FIG. 14. Variation of the total horizontal force on the interior cylinder $v s$. the ratio $a / b$ at different $k_{y}\left(b=10 \mathbf{m}, k_{x}=0.1 \mathbf{m}^{-1}\right)$. 


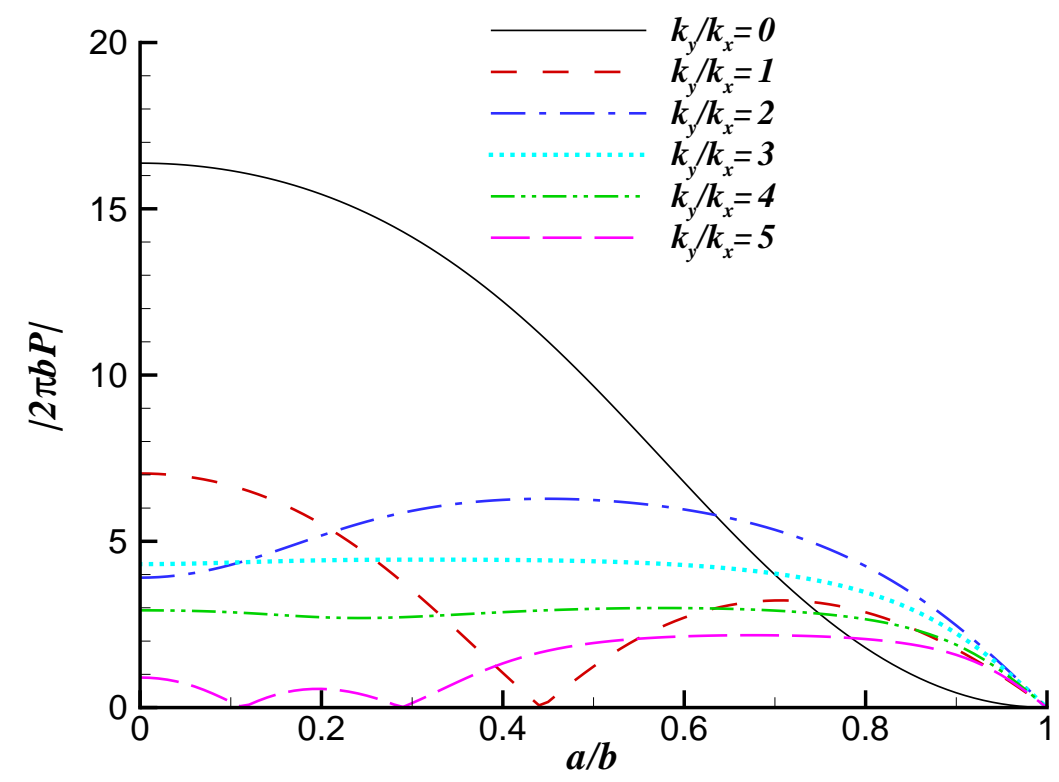

FIG. 15. Variation of the total horizontal force on the exterior cylinder $v s$. the ratio $a / b$ at different $k_{y}\left(b=10 \mathbf{m}, k_{x}=0.1 \mathbf{m}^{-1}\right)$. 


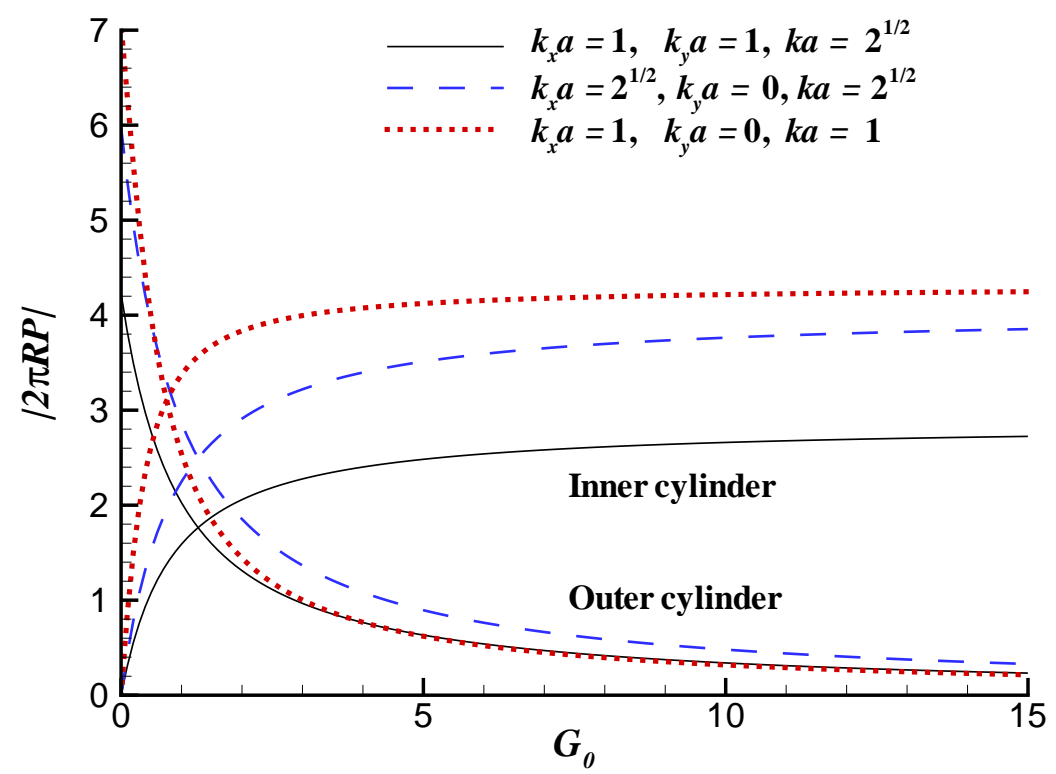

FIG. 16. Variations of the total horizontal forces of the cylinders $v s$. the porouseffect parameter $G_{0}(b / a=2$ and $a=1 \mathbf{m})$. 
Co-amplitude: Plane waves $\left(k_{x} a=1, k_{y} a=0\right)$

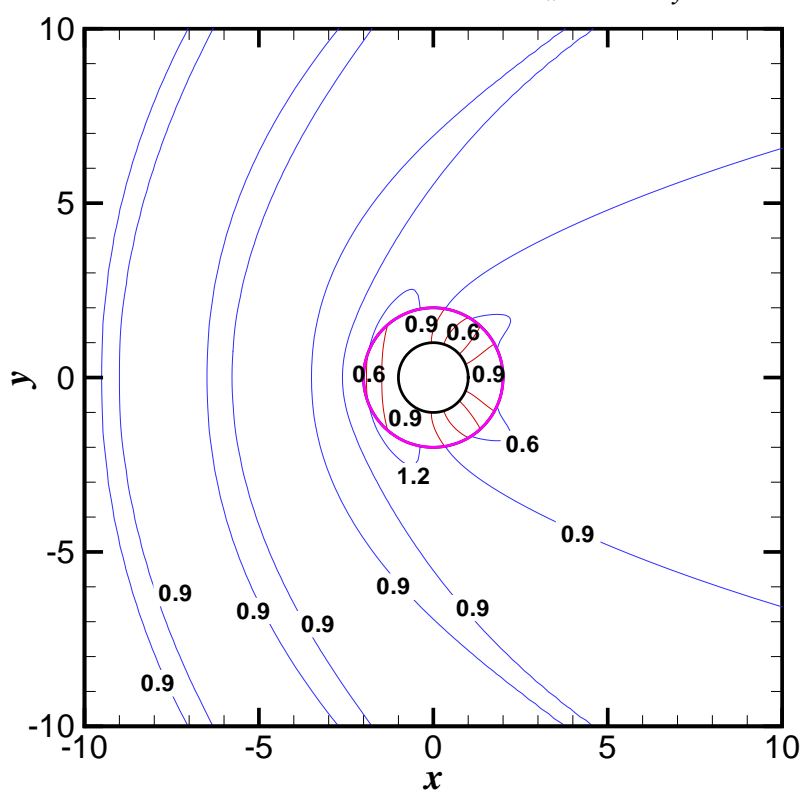

Co-amplitude: Short-crested waves $\left(k_{x} a=1, k_{y} a=1\right)$

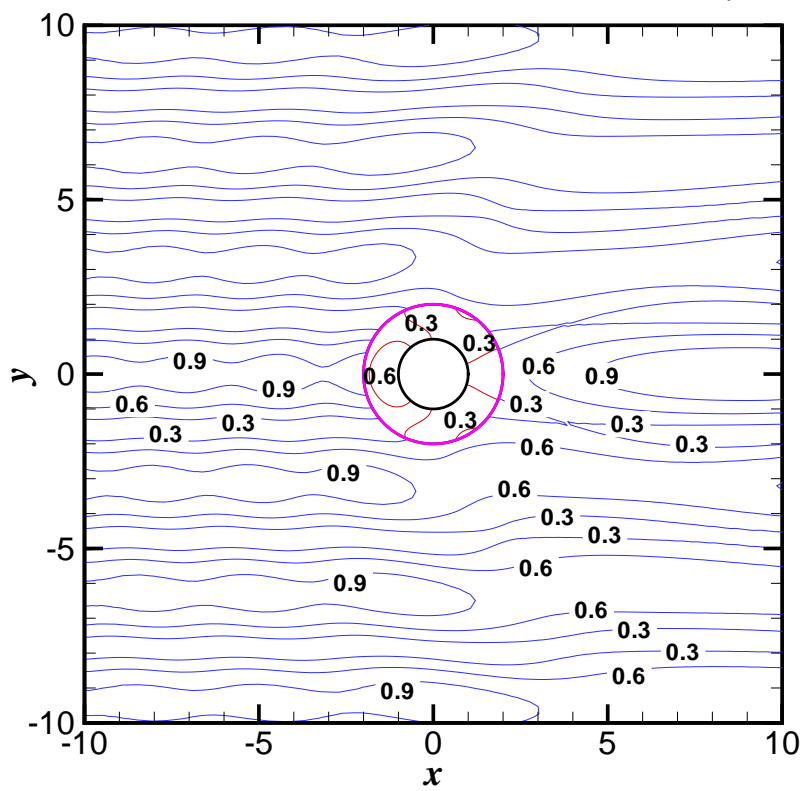

Co-amplitude: Standing waves $\left(k_{x} a=0, k_{y} a=1\right)$

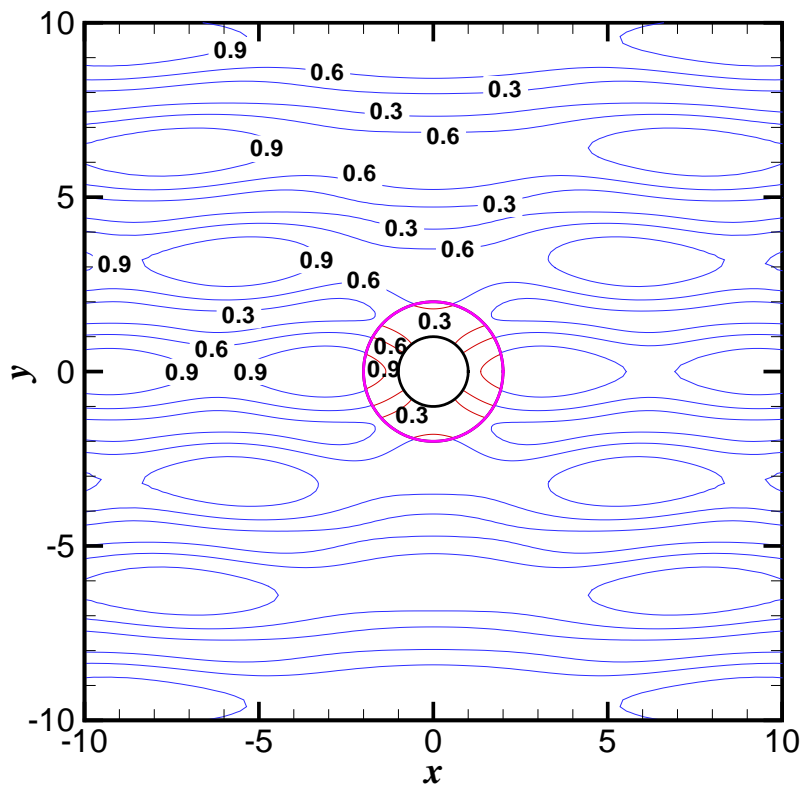

Co-phase: Plane waves $\left(k_{x} a=1, k_{y} a=0\right)$

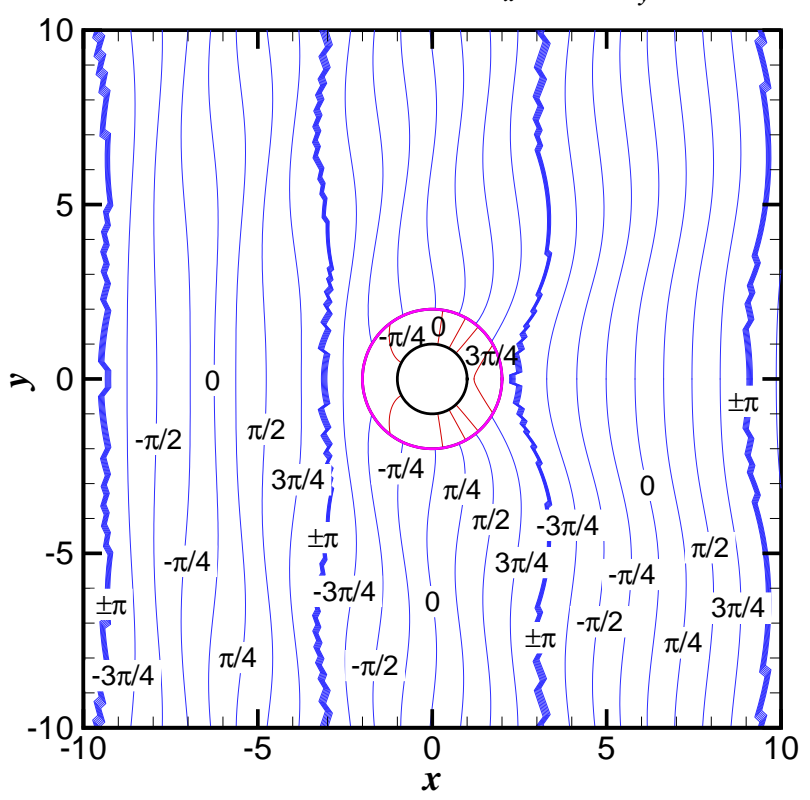

Co-phase: Short-crested waves $\left(k_{x} a=1, k_{y} a=1\right)$

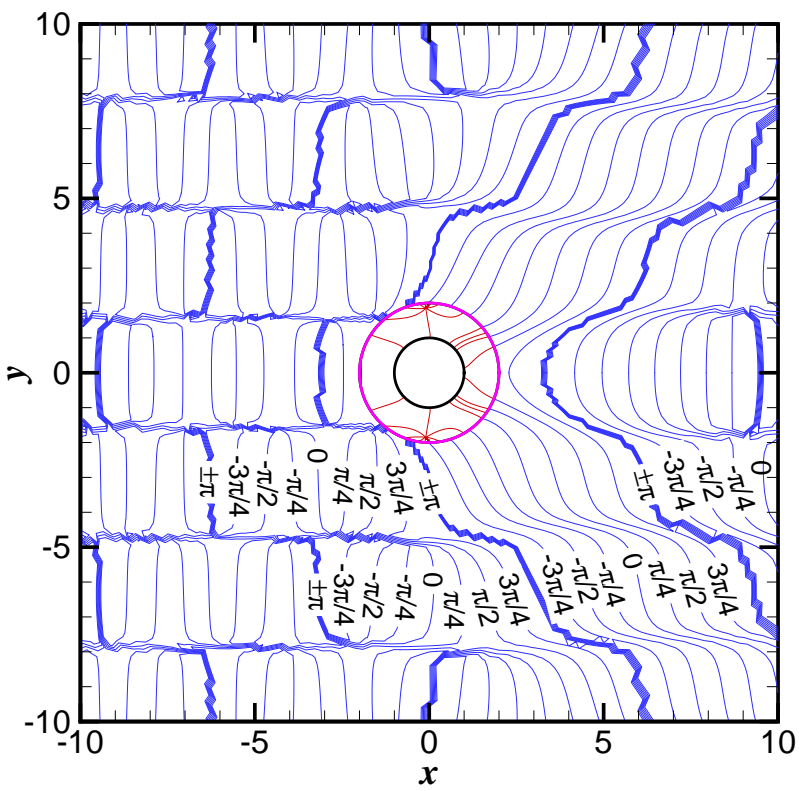

Co-phase: Standing waves $\left(k_{x} a=0, k_{y} a=1\right)$

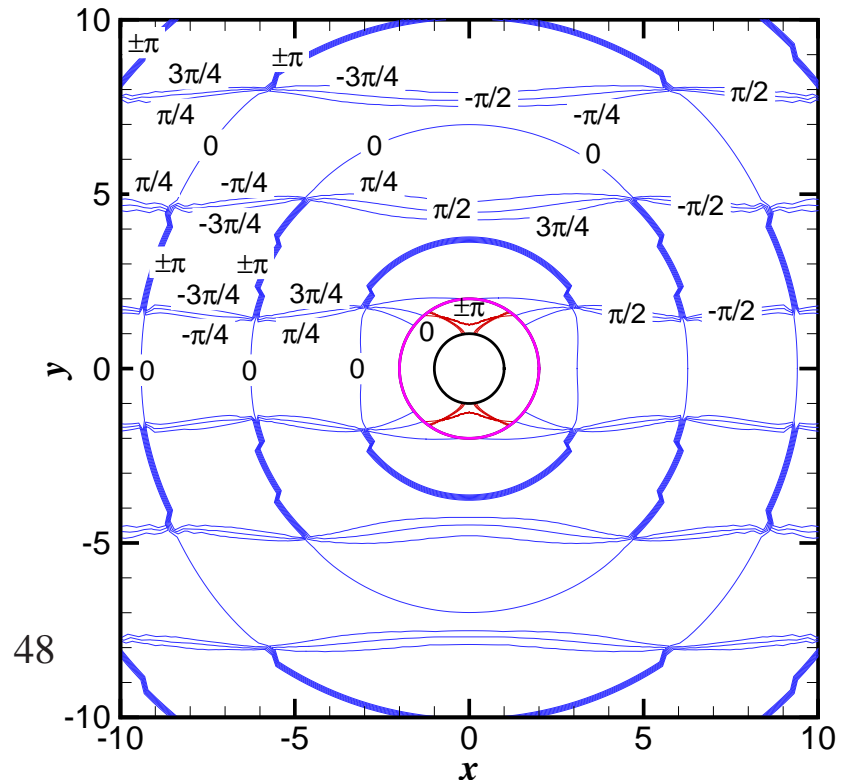

FIG. 17. Equi-amplitude contours (left) and Equi-phase contours (right) for the incident plane, short-crested, and standing waves, respectively. 